\title{
Harmonic metrics on Higgs sheaves and uniformization of varieties of general type
}

\author{
Daniel Greb ${ }^{1} \cdot$ Stefan Kebekus ${ }^{2,3} \cdot$ Thomas Peternell $^{4} \cdot$ Behrouz Taji $^{5}$
}

Received: 6 April 2018 / Revised: 4 July 2019 / Published online: 18 September 2019

(c) The Author(s) 2019

\begin{abstract}
We prove a criterion for the existence of harmonic metrics on Higgs bundles that are defined on smooth loci of klt varieties. As one application, we resolve the quasiétale uniformisation problem for minimal varieties of general type to obtain a complete numerical characterisation of singular quotients of the unit ball by discrete, co-compact groups of automorphisms that act freely in codimension one. As a further application, we establish a nonabelian Hodge correspondence on smooth loci of klt varieties.
\end{abstract}

Mathematics Subject Classification 32Q30 $14 \mathrm{E} 20 \cdot 14 \mathrm{E} 30 \cdot 53 \mathrm{C} 07$

\section{Contents}

1 Introduction . . . . . . . . . . . . . . . . . . . . . . . 1062

2 Notation and elementary facts . . . . . . . . . . . . . . . . . . . . . . 1065

Part I. Existence of harmonic bundle structures . . . . . . . . . . . . . . . . . . . . . . . . . . 1068

3 Harmonic bundles . . . . . . . . . . . . . . . . . . . . . . . . . . . . 1068

4 Higgs bundles and Higgs sheaves . . . . . . . . . . . . . . . . . . . . . . . . . . 1075

5 Existence of harmonic structures . . . . . . . . . . . . . . . . . . . . . . . . 1078

Part II. Applications . . . . . . . . . . . . . . . . . . . . . . . . . . . . . . . . . . . . . . . . . . 1084

6 Nonabelian Hodge correspondences for smooth loci . . . . . . . . . . . . . . . . . . . . . . . 1084

7 Proof of Theorem 1.5, uniformisation for minimal varieties . . . . . . . . . . . . . . . 1086

8 Positivity in the sheaf of reflexive differentials . . . . . . . . . . . . . . . . . . . 1088

References . . . . . . . . . . . . . . . . . . . . . . . . . . 1091

Communicated by Ngaiming Mok.

DG was partially supported by the DFG-Collaborative Research Center SFB/TR 45 "Periods, moduli spaces and arithmetic of algebraic varieties". SK gratefully acknowledges support through a joint fellowship of the Freiburg Institute of Advanced Studies (FRIAS) and the University of Strasbourg Institute for Advanced Study (USIAS). BT was partially supported by the DFG-Research Training Group GK1821. Research was partially completed while SK and TP were visiting the National University of Singapore in 2017.

Extended author information available on the last page of the article 


\section{Introduction}

\subsection{Main result of this paper}

The core notion of nonabelian Hodge theory, as pioneered by Corlette, Donaldson, Hitchin, and Simpson, is certainly that of a harmonic bundle. Most (if not all) important results of this theory depend on existence results for harmonic metrics in bundles over projective manifolds, which are usually established using highly non-trivial analytic methods.

In view of the minimal model program, it is clear that these results should be studied in the more general context of varieties with terminal or canonical singularities or even for klt (= Kawamata log terminal) varieties. In this context, extending Simpson's theory [37] from smooth projective manifolds to minimal models, the paper [9] established a natural equivalence between the category of local systems and the category of semistable, locally free Higgs sheaves with vanishing Chern classes on projective varieties with klt singularities

Theorem 1.1 [9, Thm. 1.1] Let $X$ be a projective, klt variety. Then, there exists an equivalence between the category of local systems on $X$ and the category of semistable, locally free Higgs sheaves with vanishing Chern classes on $X$.

However, for geometric applications we also need to understand (flat) locally free Higgs sheaves on the smooth locus $X_{\text {reg }}$ of a klt variety $X$ which extend to $X$ as reflexive Higgs sheaf rather than as a locally free Higgs sheaf. Thanks to the work of Simpson, Jost-Zuo, Mochizuki and others, there is by now a developed theory of harmonic bundles for non-compact, quasi-projective manifolds $X^{\circ}$, establishing the existence of a (essentially unique) harmonic metric on a given semisimple flat bundle $\left(E, \nabla_{E}\right)$ on $X^{\circ}$. In particular, $E$ inherits a holomorphic structure and a Higgs field. In this context, considering a compactification $X$ of $X^{\circ}$ by a simple normal crossing divisor, the notion of a tame and purely imaginary bundle (with respect to the compactification) play a decisive role. In case of a projective klt variety $X$ with smooth locus $X^{\circ}=X_{\text {reg }}$, the situation simplifies to some extent since $X \backslash X^{\circ}$ has codimension at least 2 , once one is willing to pay the price that $X$ is singular. Our point is that the singularities arising from the minimal model program are mild enough to still obtain a nonabelian Hodge theory on $X_{\text {reg }}$ that can be formulated in down-to-earth terms. Our main result can be seen as an existence result for harmonic metrics on bundles that are defined on smooth loci of klt varieties.

Theorem 1.2 (= Theorem 5.1 on page 14) Let $X$ be a projective klt space of dimension $n \geq 2$ and $H \in \operatorname{Div}(X)$ be ample. Let $\left(\mathscr{E}_{X_{\mathrm{reg}}}, \theta_{\mathscr{E}_{X_{\mathrm{reg}}}}\right)$ be a reflexive Higgs sheaf on $X_{\mathrm{reg}}$ and denote the reflexive extension of $\mathscr{E}_{X_{\text {reg }}}$ to $X$ by $\mathscr{E}_{X}$. Then, the following statements are equivalent.

(1.2.1) The Higgs sheaf $\left(\mathscr{E}_{X_{\mathrm{reg}}}, \theta_{\mathscr{E}_{X_{\mathrm{reg}}}}\right)$ is (poly)stable with respect to $H$ and the $\mathbb{Q}$ Chern characters satisfy

$$
\widehat{c h}_{1}\left(\mathscr{E}_{X}\right) \cdot[H]^{n-1}=0 \text { and } \widehat{c h}_{2}\left(\mathscr{E}_{X}\right) \cdot[H]^{n-2}=0 .
$$


(1.2.2) The sheaf $\mathscr{E}_{X_{\mathrm{reg}}}$ is locally free, $\left(\mathscr{E}_{X_{\mathrm{reg}}}, \theta_{\mathscr{E}_{X_{\mathrm{reg}}}}\right)$ is induced by a tame, purely imaginary harmonic bundle whose associated flat bundle is (semi)simple.

Remark 1.3 The symbols $\widehat{c h} \bullet$ that appear in Theorem 1.2 denote $\mathbb{Q}$-Chern characters, which exist because klt varieties have quotient singularities in codimension two. We refer to Sect. 2.7 and [10, Sect. 1.7] for a discussion, and to [8, Sect. 3] for a proper definition.

We illustrate the usefulness of Theorem 1.2 with two applications.

\subsection{Application: a nonabelian Hodge correspondence for local systems on $X_{\text {reg }}$}

The first application pertains to the nonabelian Hodge correspondence. As already mentioned, Theorem 1.1 extends the classic correspondence to projective varieties with klt singularities, in particular to minimal models of varieties of general type.

As one immediate consequence of Theorem 1.2, we find that our singular varieties exhibit two nonabelian Hodge correspondences, one linking local systems on the singular space $X$ with locally free Higgs sheaves there, and one linking local systems on the smooth locus $X_{\text {reg }}$ with Higgs sheaves that may acquire certain singularities along the singular locus of $X$. One of the key features of the theory is that the two correspondences coincide after passing to a finite quasi-étale cover $Y \rightarrow X$. We refer to reader to Sect. 6 for precise formulations.

\subsection{Application: quasi-étale uniformisation}

One of the cornerstones in the geometry of manifolds of general type is certainly the Miyaoka-Yau inequality. In its classical form, it states that

$$
\left(2(n+1) \cdot c_{2}(X)-n \cdot c_{1}(X)^{2}\right) \cdot\left[K_{X}\right]^{n-2} \geq 0,
$$

provided the canonical bundle $K_{X}$ of the $n$-dimensional projective manifold $X$ is ample. In case of equality, it is a consequence of the existence of a Kähler-Einstein metric on $X$, proven by Aubin and Yau, that the universal cover of $X$ is the unit ball in $\mathbb{C}^{n}$.

Again, in view of the minimal model program it is clear that these results should be studied in the more general context of varieties with terminal, canonical, or even klt singularities. In this context, the Miyaoka-Yau inequality has been generalised as follows; the formulation again uses $\mathbb{Q}$-Chern classes as constructed in [8, Sect. 3].

Theorem 1.4 (QQ-Miyaoka-Yau inequality, [8, Thm. 1.1] or [10, Thm. 1.5]) Let $X$ be an $n$-dimensional, projective, klt variety whose canonical class is big and nef. Then,

$$
\left(2(n+1) \cdot \widehat{c}_{2}\left(\mathscr{T}_{X}\right)-n \cdot \widehat{c}_{1}\left(\mathscr{T}_{X}\right)^{2}\right) \cdot\left[K_{X}\right]^{n-2} \geq 0
$$

The reader is encouraged to also have a look at [14], where related inequalities for pairs are discussed. 
Our new quasi-étale uniformisation result for varieties of general type may then be formulated as follows.

Theorem 1.5 (Quasi-étale uniformisation by the unit ball) Let $X$ be an $n$-dimensional, projective, klt variety whose canonical class is big and nef. If equality holds in the $\mathbb{Q}$-Miyaoka-Yau inequality, i.e.,

$$
\left(2(n+1) \cdot \widehat{c}_{2}\left(\mathscr{T}_{X}\right)-n \cdot \widehat{c}_{1}\left(\mathscr{T}_{X}\right)^{2}\right) \cdot\left[K_{X}\right]^{n-2}=0,
$$

then the canonical model of $X$ admits a quasi-étale Galois cover $Y \rightarrow X_{\mathrm{can}}$ by a projective manifold $Y$ whose universal cover is the unit ball.

We refer to Sect. 2.2 for the definition of "quasi-étale" and for further references.

Corollary 1.6 (Chern class equality forces quotient singularities) In the setting of Theorem 1.4 , equality in the $\mathbb{Q}$-Miyaoka-Yau Inequality implies that $X_{\mathrm{can}}$ has at worst quotient singularities.

Remark 1.7 Theorem 1.5 was shown by the authors in [8] under the additional assumption that the variety $X$ be non-singular in codimension two; this technical condition allowed us to use a significantly simpler argument.

The canonical models that appear in Theorem 1.5 are themselves quotients of the unit ball. The following characterisation is a minor generalisation of [8, Thm. 1.3]; the proof given in [8, Sect. 9.1] applies nearly verbatim and is therefore omitted.

Theorem 1.8 (Characterisation of singular ball quotients) Let $X$ be a normal, irreducible, compact, complex space of dimension $n$. Then, the following statements are equivalent.

(1.8.1) The space $X$ is of the form $\mathbb{B}^{n} / \widehat{\Gamma}$ for a discrete, co-compact subgroup $\widehat{\Gamma}<$ $\operatorname{Aut}_{\mathscr{O}}\left(\mathbb{B}^{n}\right)$ whose action on $\mathbb{B}^{n}$ is fixed-point free in codimension one.

(1.8.2) The space $X$ is of the form $Y / G$, where $Y$ is a smooth ball quotient, and $G$ is a finite group of automorphisms of $Y$ whose action is fixed-point free in codimension one.

(1.8.3) The space $X$ is projective and klt, the canonical divisor $K_{X}$ is ample, and we have equality in (1.5.1).

The reader is referred to [8, Sect. 10] for a discussion of the expectations regarding quotients of the ball by properly discontinuous group actions having fixed points in codimension one.

\subsection{Structure of the paper}

Section 2 gathers notation, known results and global conventions that will be used throughout the paper. 


\section{Part I}

Part I of this paper begins in Sect. 3 with a review of Mochizuki's theory of tame and purely imaginary harmonic bundles on quasi-projective varieties and discusses them in the particular setting where the quasi-projective variety is the smooth locus of a klt variety. Section 4 briefly reviews the somewhat delicate notion of a Higgs sheaf on a singular space, and discusses stability of Higgs bundles that are defined on the the smooth locus of a klt variety only. The core of Part I is, however, Sect. 5 where the the central existence result for harmonic structures is shown.

\section{Part II}

Part II of this paper concerns applications. Section 6 shows in brief how existence of harmonic structures leads to nonabelian Hodge correspondences pertaining to local systems on the smooth locus of a klt space. Section 7 proves our main result on quasiétale uniformisation, Theorem 1.5. Section 8 studies singular ball quotients, asking what positivity one might expect in $\Omega_{X}^{[1]}$, and what hyperbolicity properties might hold in the underlying spaces.

\section{Notation and elementary facts}

\subsection{Global conventions}

Throughout the present paper, all varieties and schemes will be defined over the complex numbers. We follow the notation used in the standard reference books $[15,20]$, with the exception that klt pairs are assumed to have an effective boundary divisor, see Sect. 2.6 below.

A morphism of vector bundles is always assumed to have constant rank. Notation introduced in our previous papers [8,9], will briefly be recalled before it is used.

Throughout the paper, we will freely switch between the algebraic and analytic context if no confusion is likely to arise; sheaves on quasi-projective varieties will always be algebraic.

\subsection{Varieties, subsets, morphisms}

In line with the notation used in [15], varieties are always assumed to be irreducible. The following will be used for notational convenience.

Notation 2.1 (Big and small subsets) Let $X$ be a normal, quasi-projective variety. A closed subset $Z \subset X$ is called small if $\operatorname{codim}_{X} Z \geq 2$. An open subset $U \subseteq X$ is called big if $X \backslash U$ is small.

Galois morphisms appear prominently in the literature, but their precise definition is not consistent. We will use the following definition, which does not ask Galois morphisms to be étale. 
Definition 2.2 (Covers and covering maps, Galois morphisms) A cover or covering map is a finite, surjective morphism $\gamma: X \rightarrow Y$ of normal, quasi-projective varieties. The covering map $\gamma$ is called Galois if there exists a finite group $G \subset \operatorname{Aut}(X)$ such that $\gamma$ is isomorphic to the quotient map.

As pointed out in the introduction, quasi-étale morphisms feature prominently in this paper. We recall the definition and refer the reader to [8, Def. 2.11] for a more detailed discussion.

Definition 2.3 (Quasi-étale morphisms) A morphism $f: X \rightarrow Y$ between normal varieties is called quasi-étale if $f$ is of relative dimension zero and étale in codimension one. In other words, $f$ is quasi-étale if $\operatorname{dim} X=\operatorname{dim} Y$ and if there exists a closed, subset $Z \subseteq X$ of codimension $\operatorname{codim}_{X} Z \geq 2$ such that $\left.f\right|_{X \backslash Z}: X \backslash Z \rightarrow Y$ is étale.

\subsection{Nef sheaves}

While positivity notions for vector bundles are well-established in the literature, we will need these notions also for coherent sheaves.

Definition 2.4 (Nef and ample sheaves, [1]) Let $X$ be a normal, projective variety and let $\mathscr{S} \neq 0$ be a non-trivial coherent sheaf on $X$, not necessarily locally free. We call $\mathscr{S}$ ample (resp. nef) if the locally free sheaf $\mathscr{O}_{\mathbb{P}(\mathscr{S})}(1) \in \operatorname{Pic}(\mathbb{P}(\mathscr{S}))$ is ample (resp. nef).

We refer the reader to [12] for the definition of $\mathbb{P}(\mathscr{S})$, and to [1, Sect. 2 and Thm. 2.9] for a more detailed discussion of amplitude and for further references. We mention a few elementary facts without proof.

Fact 2.5 (Nef and ample sheaves) Let $X$ be a normal, projective variety.

(2.5.1) Ample sheaves on $X$ are nef.

(2.5.2) A direct sum of sheaves on $X$ if nef iff every summand is nef.

(2.5.3) Pull-backs and quotients of nef sheaves are nef.

(2.5.4) A sheaf $\mathscr{E}$ is nef on $X$ if and only if for every smooth curve $C$ and every morphism $\gamma: C \rightarrow X$, the pull-back $\gamma^{*} \mathscr{E}$ is nef.

\subsection{Connections on complex vector bundles}

Connections on complex vector bundles play a prominent role in this paper. We recall two elementary facts that will become relevant later.

Fact 2.6 (Extension of bundles with connection) Let $M$ be a $\mathcal{C}^{\infty}$-manifold and $M^{\circ} \subseteq$ $M$ an open subset. Write $\widehat{\pi}_{1}$ for the profinite completion of the fundamental group, and let $\widehat{\rho}: \widehat{\pi}_{1}\left(M^{\circ}\right) \rightarrow \widehat{\pi}_{1}(M)$ be the natural morphism induced by the inclusion. If $\widehat{\rho}$ is isomorphic, then any flat, complex bundle $\left(E^{\circ}, \nabla_{E^{\circ}}\right)$ on $M^{\circ}$ admits an extension to a flat, complex bundle $\left(E, \nabla_{E}\right)$ on $M$. The extension is unique up to canonical isomorphism. 
If $\gamma: X \rightarrow Y$ is a ramified Galois cover of complex manifolds, if $E$ is a smooth, complex bundle over $Y$ and $h$ a smooth, Galois-invariant Hermitian metric on $\gamma^{*} E$, it is generally not true that $h$ comes from a smooth metric on $Y$. In contrast, the following result asserts that flat connections in $\gamma^{*} E$ do indeed descend once they are invariant. This is probably known to experts. The arXiv version of this paper includes a full proof.

Proposition 2.7 (Descent of $G$-invariant, flat connections) Let $\gamma: X \rightarrow Y$ be a Galois cover of complex manifolds, with Galois group $G$. Let $E_{Y}$ be a smooth, complex bundle over $Y$ and let $\nabla_{X}$ be a flat, G-invariant connection on $E_{X}:=\gamma^{*} E_{Y}$. Then, there exists a flat connection $\nabla_{Y}$ on $E_{Y}$ such that $\nabla_{X}=\gamma^{*} \nabla_{Y}$.

\subsection{Higgs sheaves}

Let $X$ be a normal variety or normal complex space. Following the notation introduced in [8, Def. 5.1], a Higgs sheaf is a pair $(\mathscr{E}, \theta)$ of a coherent sheaf $\mathscr{E}$ of $\mathscr{O}_{X}$-modules, together with an $\mathscr{O}_{X}$-linear sheaf morphism $\theta: \mathscr{E} \rightarrow \mathscr{E} \otimes \Omega_{X}^{[1]}$, called Higgs field, such that the induced morphism $\mathscr{E} \rightarrow \mathscr{E} \otimes \Omega_{X}^{[2]}$ vanishes. We refer the reader to [8, Sect. 5] for related notions, including the definition of a Higgs $G$-sheaf, and slope stability of Higgs sheaves with respect to nef divisor classes.

\subsubsection{Categories used in the nonabelian Hodge correspondence}

If $X$ is a projective normal variety, we often consider locally free Higgs sheaves $(\mathscr{E}, \theta)$ on $X$ having the property that there exists an ample divisor $H \in \operatorname{Div}(X)$ such that

- the Higgs sheaf $(\mathscr{E}, \theta)$ is semistable with respect to $H$, and

- the Chern characters of $\mathscr{E}$ satisfy $\operatorname{ch}_{1}(\mathscr{E}) \cdot[H]^{n-1}=\operatorname{ch}_{2}(\mathscr{E}) \cdot[H]^{n-2}=0$.

These sheaves form a category, which plays a central role in the nonabelian Hodge correspondence for klt spaces, [9, Sect. 3.1]. We denote this category by Higgs ${ }_{X}$.

\subsection{KLT spaces and $\mathbb{Q}$-Chern classes}

A klt pair $(X, \Delta)$ consists of a normal variety $X$ and a Weil $\mathbb{Q}$-divisor $\Delta=\sum_{i} a_{i} D_{i}$ with $a_{i} \in \mathbb{Q} \cap(0,1)$ such that $K_{X}+\Delta$ is $\mathbb{Q}$-Cartier and such that $\operatorname{discrep}(X, \Delta)>-1$, where the discrepancy discrep $(X, \Delta)$ is defined in [20, Def. 2.28] using [20, Def. 2.25]. In contrast to [20, Def. 2.34] we do not allow non-effective boundary divisors $\Delta$.

Definition 2.8 A normal, quasi-projective variety $X$ is called klt space if there exists an effective $\mathbb{Q}$-divisor $\Delta$ that makes the pair $(X, \Delta)$ klt.

\subsection{Q-Chern classes}

Two-dimensional klt spaces are known to have quotient singularities. Their geometry can then be studied using generalised Chern classes, known as $\mathbb{Q}$-Chern classes or 
orbifold Chern classes. In higher dimensions, a klt space $X$ does not necessarily have quotient singularities. However, once one removes a suitable subset $Z \subseteq X$ of codimension three, only quotient singularities remain and $X \backslash Z$ can be equipped with the structure of a $\mathbb{Q}$-variety that admits a global, Cohen-Macaulay cover. In particular, following Mumford's fundamental paper [30], Chern classes can be defined. Since $\operatorname{codim} Z=3$, this allows us to construct on any $n$-dimensional klt space $X$ useful intersection products with first and second $\mathbb{Q}$-Chern classes of any reflexive sheaf on $\mathscr{E}_{X}$. The associated symmetric, $\mathbb{Q}$-multilinear forms will be written as follows,

$$
\begin{array}{lll}
\widehat{c}_{1}(\mathscr{E}): N^{1}(X)_{\mathbb{Q}}^{\times(n-1)} \rightarrow \mathbb{Q}, & \left(\alpha_{1}, \ldots, \alpha_{n-1}\right) \mapsto \widehat{c}_{1}(\mathscr{E}) \cdot \alpha_{1} \cdots \alpha_{n-1} \\
\widehat{c}_{1}(\mathscr{E})^{2}: N^{1}(X)_{\mathbb{Q}}^{\times(n-2)} \rightarrow \mathbb{Q}, & \left(\alpha_{1}, \ldots, \alpha_{n-2}\right) \mapsto \widehat{c}_{1}(\mathscr{E})^{2} \cdot \alpha_{1} \cdots \alpha_{n-2} \\
\widehat{c}_{2}(\mathscr{E}): N^{1}(X)_{\mathbb{Q}}^{\times(n-2)} \rightarrow \mathbb{Q}, & \left(\alpha_{1}, \ldots, \alpha_{n-2}\right) \mapsto \widehat{c}_{2}(\mathscr{E}) \cdot \alpha_{1} \cdots \alpha_{n-2} .
\end{array}
$$

For notational convenience, we also consider $\mathbb{Q}$-Chern characters

$$
\widehat{c h}_{1}\left(\mathscr{E}_{X}\right)=\widehat{c}_{1}\left(\mathscr{E}_{X}\right) \text { and } \widehat{c h}_{2}\left(\mathscr{E}_{X}\right):=\frac{1}{2}\left(\widehat{c}_{1}\left(\mathscr{E}_{X}\right)^{2}-2 \cdot \widehat{c}_{2}\left(\mathscr{E}_{X}\right)\right)
$$

We refer the reader to [8, Sect. 3] for a full account of these matters. Here, we only recall the behaviour of $\mathbb{Q}$-Chern classes under quasi-étale covers.

Lemma 2.9 (Behaviour under quasi-étale covers, [8, Lem. 3.16]) If $\gamma: Y \rightarrow X$ is a quasi-étale morphism between projective klt spaces, then the following equalities hold for all reflexive sheaves $\mathscr{E}$ on $X$ and all numerical classes $\alpha_{1}, \ldots, \alpha_{n-1} \in N^{1}(X)_{\mathbb{Q}}$,

$$
\widehat{c}_{1}\left(\gamma^{[*]} \mathscr{E}\right) \cdot\left(\gamma^{*} \alpha_{1}\right) \cdots\left(\gamma^{*} \alpha_{n-1}\right)=(\operatorname{deg} \gamma) \cdot \widehat{c}_{1}(\mathscr{E}) \cdot \alpha_{1} \cdots \alpha_{n-1},
$$

where $\gamma^{[*]} \mathscr{E}:=\left(\gamma^{*} \mathscr{E}\right)^{* *}$. Analogous statements hold for $\widehat{c}_{1}\left(\gamma^{[*]} \mathscr{E}\right)^{2}$ and $\widehat{c_{2}}\left(\gamma^{[*]} \mathscr{E}\right)$.

Remark 2.10 The sheaf $\gamma^{[*]} \mathscr{E}$ in Lemma 2.9 is usually not isomorphic to the pull-back $\gamma^{*} \mathscr{E}$.

\section{Part I. Existence of harmonic bundle structures}

\section{Harmonic bundles}

Harmonic bundles are key tools in nonabelian Hodge theory that provide the link between flat structures and Higgs fields. We briefly recall the definition, explain relevant properties, and recall the notion of "tameness" that is used to establish a good theory in non-compact, compactifiable situations. Finally, we prove a boundedness result for Higgs bundles admitting a tame and purely imaginary harmonic structure.

Fact and Definition 3.1 (Harmonic bundle, cf. [33, Sect. 1]) Let $M$ be a complex manifold. Consider a tuple $\mathbb{E}=(E, \bar{\partial}, \theta, h)$ comprised of the following data. 
- A holomorphic vector bundle $(E, \bar{\partial})$ and a Hermitian metric $h$ on $E$.

- A Higgs field $\theta: \mathscr{E} \rightarrow \mathscr{E} \otimes \Omega_{X}^{1}$, where $\mathscr{E}=\operatorname{ker} \bar{\partial}$ is the sheaf of holomorphic sections.

By minor abuse of notation, we will also write $\theta$ for the induced $\mathscr{A}^{0}$-linear morphism $\theta: \mathscr{A}^{0}(E) \rightarrow \mathscr{A}^{1,0}(E)$. Let $\theta^{h}: \mathscr{A}^{0}(E) \rightarrow \mathscr{A}^{0,1}(E)$ be the adjoint ${ }^{1}$ of $\theta$ with respect to the metric $h$, and let $\partial$ be the $(1,0)$-part of the unique Chern-connection compatible with both the metric $h$ and the complex structure $\bar{\partial}$. Then,

$$
\nabla_{\mathbb{E}}:=\partial+\bar{\partial}+\theta+\theta^{h}
$$

is a connection. The tuple $\mathbb{E}$ is called a harmonic bundle if $\nabla_{\mathbb{E}}$ is flat.

Notation 3.2 (Flat bundles and local systems associated with harmonic bundles) Given a harmonic bundle $\mathbb{E}=(E, \bar{\partial}, \theta, h)$ as in Fact and Definition 3.1, we denote the associated flat bundle by $\left(E, \nabla_{\mathbb{E}}\right)$ and write $E \in \mathrm{LSys}_{M}$ for the local system.

Notation 3.3 (Sheaves admitting a harmonic bundle structure) Let $M$ be a complex manifold.

- Given a locally free sheaf $\mathscr{E}$ on $M$ with associated holomorphic bundle $(E, \bar{\partial})$, we say that $\mathscr{E}$ admits a harmonic bundle structure if there exists a harmonic bundle of the form $(E, \bar{\partial}, \theta, h)$.

- Given a locally free Higgs sheaf $(\mathscr{E}, \theta)$ on $M$ with associated holomorphic bundle $(E, \bar{\partial})$, we say that $(\mathscr{E}, \theta)$ admits a harmonic bundle structure if there exists a harmonic bundle of the form $(E, \bar{\partial}, \theta, h)$.

- We say that a flat bundle $(E, \nabla)$ admits a harmonic bundle structure if there exists a harmonic bundle $\mathbb{E}=(E, \bar{\partial}, \theta, h)$ such that $\nabla=\nabla_{\mathbb{E}}$.

If $X$ is a smooth, quasi-projective manifold and $\mathscr{E}$ a locally free sheaf on $X$, we say that $\mathscr{E}$ admits a harmonic bundle structure if its analytification $\mathscr{E} a n$ on the complex manifold $X^{a n}$ admits a harmonic bundle structure. Analogously for algebraic Higgs bundles $(\mathscr{E}, \theta)$ on $X$.

Remark 3.4 In the setup of Notation 3.3, let $N$ be a complex submanifold of $M$. Assume that $(\mathscr{E}, \theta)$ admits a harmonic bundle structure. Then, an easy local computation shows that the locally free Higgs sheaf $\left.(\mathscr{E}, \theta)\right|_{N}$ admits a harmonic bundle structure given by restriction.

\subsection{Tame and purely imaginary bundles}

In order to study Higgs bundles on quasi-projective, non-projective varieties, we consider "tame" harmonic bundles. These are harmonic bundles on the complement of a divisor whose growth near the divisor is sufficiently controlled.

\footnotetext{
$\overline{1 \text { In local coordinates, if } \theta=\sum_{k}} \theta_{k} d z_{k}$, then $\theta^{h}=\sum_{k} \theta_{k}^{*} d \bar{z}_{k}$, where $\theta_{k}^{*}$ is the adjoint of $\theta_{k}$ with respect to the metric $h$.
} 


\subsubsection{Basic definitions}

The following is not Simpson's original definition of "tameness", but is equivalent to it.

Definition 3.5 (Tame harmonic bundle, [27, Sect. 22.1 and Lem. 22.1]) Let $M$ be a complex manifold, let $D \subset M$ be a divisor with simple normal crossings, and let $\mathbb{E}=(E, \bar{\partial}, \theta, h)$ be a harmonic bundle on $M \backslash D$. The harmonic bundle $\mathbb{E}$ is called tame with respect to $(M, D)$ if there exists a locally free sheaf $\mathscr{E}_{M}$ on $M$, a sheaf morphism

$$
\theta_{M}: \mathscr{E}_{M} \rightarrow \mathscr{E}_{M} \otimes \Omega_{M}^{1}(\log D)
$$

and an isomorphism $\left.\mathscr{E}_{M}\right|_{M \backslash D} \cong \mathscr{E}$ that identifies $\left.\theta_{M}\right|_{M \backslash D}$ with $\theta$. We call $\left(\mathscr{E}_{M}, \theta_{M}\right)$ an extension of $(\mathscr{E}, \theta)$.

Fact and Definition 3.6 (Purely imaginary bundles, [27, Lem. 22.2]) In the setup of Definition 3.5, assume that $(E, \bar{\partial}, \theta, h)$ is tame, and let $\left(\mathscr{E}_{M}, \theta_{M}\right)$ be an extension of $(\mathscr{E}, \theta)$. If $D_{i} \subset D$ is any component and $x \in D_{i}$ is any point, consider the residue and its restriction to $x$,

$$
\operatorname{res}_{D_{i}} \theta_{M} \in \operatorname{End}\left(\left.\mathscr{E}_{M}\right|_{D_{i}}\right) \text { and }\left.\left(\operatorname{res}_{D_{i}} \theta_{M}\right)\right|_{x} \in \operatorname{End}\left(\left.\mathscr{E}_{M}\right|_{x}\right)
$$

Then, then the set of eigenvalues of $\left.\left(\operatorname{res}_{D_{i}} \theta_{M}\right)\right|_{x}$ is independent of the choice of $\left(\mathscr{E}_{M}, \theta_{M}\right)$.

The harmonic bundle $\left(E, \bar{\partial}_{E}, \theta, h\right)$ is called purely imaginary with respect to $(M, D)$ if all eigenvalues of the residues of $\theta_{M}$ along the irreducible components of $D$ are purely imaginary for one (equivalently any) extension $\left(\mathscr{E}_{M}, \theta_{M}\right)$ of $(\mathscr{E}, \theta)$.

It is important to notice that the notion of tame purely imaginary bundles does not depend on the compactification.

Fact and Definition 3.7 (Tame and purely imaginary bundles on quasi-projective varieties, [27, Lem. 25.29] and [26, Cor. 8.7]) Let X be a smooth, quasi-projective variety and let $\mathbb{E}:=(E, \bar{\partial}, \theta, h)$ be a harmonic bundle on $X^{\text {an }}$. Let $\bar{X}_{1}$ and $\bar{X}_{2}$ be two smooth, projective compactifications of $X$ such that $D_{\bullet}:=\bar{X}_{\bullet} \backslash X$ are snc divisors. Then, $\mathbb{E}$ is tame and purely imaginary with respect to $\left(\bar{X}_{1}, D_{1}\right)$ if and only if $\mathbb{E}$ is tame and purely imaginary with respect to $\left(\bar{X}_{2}, D_{2}\right)$. We can therefore speak about tame and purely imaginary harmonic bundles on $X^{a n}$.

Remark 3.8 (Automatic algebraicity I) In the setup of Fact and Definition 3.7, setting as usual $\mathscr{E}:=$ ker $\bar{\partial}$, we may apply Serre's GAGA to the extension of $\mathscr{E}$ to a smooth projective simple normal crossings compactification of $X$ (as in Definition 3.5) to see that the holomorphic Higgs sheaf $(\mathscr{E}, \theta)$ on $X^{a n}$ can be endowed with an algebraic structure. 
Remark and Notation 3.9 (Uniqueness of the algebraic structure) We will often consider the setup of Fact and Definition 3.7 in a situation where the smooth variety $X$ is a big open subset of a normal projective variety $\bar{X}$. If $\mathscr{E}$ is any locally free sheaf on $X^{a n}$ that admits a tame and purely imaginary harmonic bundle structure, then $\mathscr{E}$ can be endowed with an algebraic structure and hence has a coherent extension to $\bar{X}^{a n}$. By [35, Thm. 1], the analytic sheaf $\mathscr{E}$ will then have a unique reflexive extension to $\bar{X}^{a n}$. It then follows from GAGA that the induced algebraic structure on $\mathscr{E}$ is unique up to isomorphism; the same holds for the Higgs field as well.

In this situation, we simply say that $\mathscr{E}$ and $(\mathscr{E}, \theta)$ are algebraic, and freely switch between the analytic and the algebraic category if no confusion seems likely.

Remark 3.10 Flat sheaves admitting a tame and purely imaginary harmonic bundle structure are necessarily semisimple, see [27, Prop. 22.15].

Notation 3.11 (Bundles admitting tame, purely imaginary harmonic structures). If $X$ is a smooth, quasi-projective variety, write

\section{TPI-locFree $X$}

for the family of (algebraic) isomorphism classes of locally free (algebraic) sheaves on $X$ that admit a tame, purely imaginary harmonic bundle structure. Write

\section{TPI-Higgs $_{X}$}

for the family of algebraic isomorphism classes of locally free Higgs sheaves on $X$ that admit a tame, purely imaginary harmonic bundle structure. Abusing notation, we write $(\mathscr{E}, \theta) \in \mathrm{TPI}^{-H_{i g g s}}$ to indicate that a given Higgs sheaf $(\mathscr{E}, \theta)$ on $X$ is locally free and admits a tame, purely imaginary harmonic bundle structure $\mathbb{E}$. If $(\mathscr{E}, \theta) \in$ TPI-Higgs $_{X}$ and if $X$ is a big open subset of a normal projective variety $\bar{X}$, we also say that $(\mathscr{E}, \theta)$ is induced by $\mathbb{E}$.

Lemma 3.12 (Flat subsheaves in tame and purely imaginary harmonic bundles) Let $X$ be a smooth, quasi-projective variety and let $\mathbb{E}=\left(E, \bar{\partial}_{E}, \theta, h\right)$ be a tame and purely imaginary harmonic bundle on $X^{\text {an }}$ with induced flat connection $\nabla_{\mathbb{E}}$. If $F \subseteq E$ is any complex subbundle that is invariant with respect to $\nabla_{\mathbb{E}}$, then $\bar{\partial}$ restricts to equip $F$ with the structure of a Higgs-invariant, holomorphic subbundle of $(E, \bar{\partial})$.

Proof. It suffices to consider the case where $F$ with its induced flat structure is irreducible. But there, the description of the tame and purely imaginary harmonic bundle $\mathbb{E}$ in [25, Lem. A.13] immediately implies that the metric complement $F^{\perp}$ of $F$ is likewise invariant with respect to $\nabla_{\mathbb{E}}$. The claim then follows from the description of the operators $\bar{\partial}$ and $\theta$ in terms of $\nabla_{\mathbb{E}}$ and $h$, cf. [37, p. 13]. The arXiv version of this paper spells out all details.

\subsubsection{Existence and uniqueness}

If $X$ is smooth and quasi-projective, then a result of Jost-Zuo [17] implies that every semisimple flat bundle on $X$ admits a tame and purely imaginary metric, which is 
essentially unique. ${ }^{2}$ We summarise the results relevant for us in the following theorem, see [25, Lem. A.13] and further references given there.

Theorem 3.13 (Existence and uniqueness of harmonic structures) Let $X$ be a smooth quasi-projective variety. Then, every semisimple flat vector bundle $\left(E, \nabla_{E}\right)$ on $X$ admits a tame, purely imaginary harmonic bundle structure $\mathbb{E}=(E, \bar{\partial}, \theta, h)$. The metric $h$ is unique up to flat automorphisms of $E$, and, as a consequence, the operators in the induced decomposition (3.1.1) are independent of the choice of such $h$.

The following consequences will be used later.

Corollary 3.14 (Higgs bundles determined by induced connection) Let X be a smooth, quasi-projective variety and let $\mathbb{E}:=\left(E, \bar{\partial}_{E}, \theta_{\mathscr{E}}, h_{E}\right)$ and $\mathbb{F}:=\left(F, \bar{\partial}_{F}, \theta_{\mathscr{F}}, h_{F}\right)$ be two tame, purely imaginary harmonic bundles on $X^{(a n)}$, with associated locally free sheaves $\mathscr{E}$ and $\mathscr{F}$. Assume that the flat bundles $\left(E, \nabla_{\mathbb{E}}\right)$ and $\left(F, \nabla_{\mathbb{F}}\right)$ are isomorphic. Then, also the corresponding holomorphic Higgs bundles are holomorphically isomorphic, $\left(\mathscr{E}, \theta_{\mathscr{E}}\right) \cong\left(\mathscr{F}, \theta_{\mathscr{F}}\right)$.

Proof. By assumption, there exists a smooth isomorphism $\Phi: E \rightarrow F$ such that $\Phi^{*} \nabla_{\mathbb{F}}=\nabla_{\mathbb{E}}$. The pull-back $\Phi^{*} \mathbb{F}=\left(E, \Phi^{*} \bar{\partial}_{F}, \Phi^{*} \theta_{\mathscr{F}}, \Phi^{*} h_{F}\right)$ will thus equip $E$ with a second tame, purely imaginary harmonic bundle structure, whose associated flat connection $\nabla_{\Phi^{*} \mathbb{F}}$ equals $\nabla_{\mathbb{E}}$. But then it follows from Theorem 3.13 that the differential operators in the two harmonic bundle structures agree: $\bar{\partial}_{E}=\Phi^{*} \bar{\partial}_{F}$ and $\theta_{\mathscr{E}}=\Phi^{*} \theta_{\mathscr{F}}$. In other words, $\Phi$ is holomorphic and induces a holomorphic isomorphism of Higgs bundles.

Remark 3.15 (Automatic algebraicity II) In the setting of Corollary 3.14, recall from Remark 3.8 that the Higgs sheaves $\left(\mathscr{E}, \theta_{\mathscr{E}}\right)$ and $\left(\mathscr{F}, \theta_{\mathscr{F}}\right)$ are in fact algebraic. If $X$ is isomorphic to a big open subset in a normal variety, then the holomorphic isomorphism given in Corollary 3.14 extends to an isomorphism between reflexive closures, and is therefore likewise algebraic.

Corollary 3.16 (Extension of harmonic bundles from hyperplanes) Let $X$ be a normal, projective variety of dimension $\operatorname{dim} X>2$, and let $H \in \operatorname{Div}(X)$ be ample. If $m \gg 0$ is large enough and $D \in|m \cdot H|$ is general, then $D$ is normal, $D_{\mathrm{reg}}=D \cap X_{\mathrm{reg}}$, and the restriction map TPI-Higgs $X_{\text {reg }} \rightarrow$ TPI-Higgs $D_{D_{\mathrm{reg}}}$ is surjective.

Proof. If $m \gg 0$ is large enough, then $|m \cdot H|$ is basepoint free, we have $D_{\text {reg }}=$ $D \cap X_{\text {reg }}$ by Bertini, and the Lefschetz hyperplane theorem for fundamental groups, [11, Thm. in Sect. II.1.2] implies that the natural morphism $\pi_{1}\left(D_{\text {reg }}\right) \rightarrow \pi_{1}\left(X_{\text {reg }}\right)$ is isomorphic. Now, assuming we are given a $\left(\mathscr{E}_{D_{\text {reg }}}, \theta_{\mathscr{E}_{D_{\text {reg }}}}\right) \in$ TPI-Higgs ${ }_{D_{\text {reg }}}$, we need to show that there exists $\left(\mathscr{F}_{X_{\text {reg }}}, \theta_{\mathscr{F}_{X_{\text {reg }}}}\right) \in$ TPI-Higgs $_{X_{\text {reg }}}$ such that

$$
\left.\left(\mathscr{E}_{D_{\text {reg }}}, \theta_{\mathscr{E}_{D_{\text {reg }}}}\right) \cong\left(\mathscr{F}_{X_{\text {reg }}}, \theta_{\mathscr{F}_{X_{\text {reg }}}}\right)\right|_{D_{\text {reg }}}
$$

To this end, choose a tame, purely imaginary harmonic bundle $\mathbb{E}_{D_{\text {reg }}}$ inducing $\left(\mathscr{E}_{D_{\text {reg }}}, \theta_{\mathscr{E}_{D_{\text {reg }}}}\right)$, which exists by assumption, and recall from Remark 3.10 that the

\footnotetext{
2 See the argument in [27, Thm. 25.28].
} 
induced local system $E_{D_{\text {reg }}} \in$ LSys $_{D_{\text {reg }}}$ is semisimple. Then, using the Lefschetz Theorem we can extend $E_{D_{\text {reg }}}$ to a semisimple local system $F_{X_{\text {reg }}} \in \mathrm{LSys}_{X_{\text {reg }}}$ in a unique manner. By the Jost-Zuo existence result for harmonic structures, Theorem 3.13, there will then exist a tame, purely imaginary harmonic bundle $\mathbb{F}_{X_{\text {reg }}}$ on $X_{\text {reg }}$ that induces $F_{X_{\text {reg }}}$. If ( $\left.\mathscr{F}_{X_{\text {reg }}}, \theta_{\mathscr{F}_{X_{\text {reg }}}}\right)$ is the associated Higgs bundle on $X_{\text {reg }}$, Corollary 3.14 together with Remark 3.15 gives the desired (algebraic) isomorphism (3.16.1).

\subsection{TPI-Harmonic bundles on klt spaces}

Let $X$ be a projective klt space. Using the nonabelian Hodge correspondence for locally free Higgs sheaves on klt spaces, [9, Thm. 3.4], we will show boundedness of the family TPI-Higgs ${ }_{X_{\text {reg }}}$. As a consequence, we will obtain in Corollary 3.20 a criterion for the existence of harmonic structures: a given Higgs bundle on $X_{\text {reg }}$ admits a harmonic structure if and only if its restriction to the smooth locus of a general hypersurface does. The proof uses the existence, for every klt space $X$, of a "maximally quasi-étale cover". This is a quasi-étale cover $\gamma: Y \rightarrow X$ such that the natural map of étale fundamental groups, $\widehat{\pi}_{1}\left(Y_{\text {reg }}\right) \rightarrow \widehat{\pi}_{1}(Y)$, is isomorphic. The existence of such a cover was established in [7, Thm. 1.5].

Proposition 3.17 (Pull-back of Higgs sheaves to maximally quasi-étale cover) Let $X$ be a projective klt space. Let $\gamma: Y \rightarrow X$ be a maximally quasi-étale cover, write $X^{\circ}:=X_{\mathrm{reg}}, Y^{\circ}:=\gamma^{-1}\left(X^{\circ}\right)$ and consider the étale morphism $\delta: Y^{\circ} \rightarrow X^{\circ}$ given as the restriction of $\gamma$. Given $\left(\mathscr{E}_{X^{\circ}}, \theta_{\mathscr{E}_{X^{\circ}}}\right) \in \mathrm{TPI}^{\circ}$ Higgs $_{X^{\circ}}$, there exists $\left(\mathscr{F}_{Y}, \theta_{\mathscr{F}_{Y}}\right) \in$ Higgs $_{Y}$ and an isomorphism

$$
\left.\left(\mathscr{F}_{Y}, \theta_{\mathscr{F}_{Y}}\right)\right|_{Y^{\circ}} \cong \delta^{*}\left(\mathscr{E}_{X^{\circ}}, \theta_{\mathscr{E}_{X^{\circ}}}\right)
$$

In particular, if $\mathscr{E}_{X}$ denotes the reflexive extension of $\mathscr{E}_{X}$ to $X$, then $\gamma^{[*]} \mathscr{E}_{X} \cong \mathscr{F}_{Y}$ is locally free and all its Chern classes vanish.

Remark and Notation 3.18 In the setting of Proposition 3.17, if $\gamma$ is Galois with group $G$, then $\left(\mathscr{F}_{Y}, \theta_{\mathscr{F}_{Y}}\right)$ carries the structure of a Higgs $G$-sheaf. Writing $\left(\mathscr{F}_{Y^{\circ}}, \theta_{\mathscr{F}_{Y^{\circ}}}\right):=$ $\left.\left(\mathscr{F}_{Y}, \theta_{\mathscr{F}_{Y}}\right)\right|_{Y^{\circ}}$, the invariant push-forward of the $G$-equivariant Higgs field $\theta_{\mathscr{F}_{Y^{\circ}}}$,

$$
\left(\delta_{*} \theta_{\mathscr{F}_{Y^{\circ}}}\right)^{G}: \underbrace{\left(\delta_{*} \mathscr{F}_{Y^{\circ}}\right)^{G}}_{\cong \mathscr{E}^{\circ}} \rightarrow \underbrace{\left(\delta_{*}\left(\mathscr{F}_{Y^{\circ}} \otimes \Omega_{Y^{\circ}}^{1}\right)\right)^{G}}_{\cong \mathscr{E}_{X^{\circ}} \otimes \Omega_{X^{\circ}}^{1} \text { since } \delta \text { is étale }}
$$

is immediately identified with the Higgs field $\theta_{\mathscr{E}_{X^{\circ}}}$. Abusing notation, we write $\left(\mathscr{E}_{X^{\circ}}, \theta_{\mathscr{E}_{X^{\circ}}}\right)=\delta_{*}\left(\mathscr{F}_{Y^{\circ}}, \theta_{\mathscr{F}_{Y^{\circ}}}\right)^{G}$ in this context.

Proof of Proposition 3.17 Write $\left(\mathscr{F}_{Y^{\circ}}, \theta_{\mathscr{F}_{Y^{\circ}}}\right):=\delta^{*}\left(\mathscr{E}_{X^{\circ}}, \theta_{\mathscr{E}_{X^{\circ}}}\right)$ and choose a tame, purely imaginary harmonic bundle structure $\mathbb{E}_{X^{\circ}}$ for $\left(\mathscr{E}_{X^{\circ}}, \theta_{\mathscr{E}_{X^{\circ}}}\right)$. Recall from [27, Lem. 25.29] that the pullback $\mathbb{F}_{Y^{\circ}}:=\delta^{*} \mathbb{E}_{X^{\circ}}$ is a tame and purely imaginary harmonic bundle structure for $\left(\mathscr{F}_{Y^{\circ}}, \theta_{\mathscr{F}_{Y^{\circ}}}\right)$. The induced local system $F_{Y^{\circ}}$ is semisimple by [27, Prop. 22.15]. The assumption that $Y$ is maximally quasi-étale implies that $F_{Y \circ}$ extends 
from $Y^{\circ}$ to a semisimple local system $F_{Y} \in \mathrm{LSys}_{Y}$ that is defined on all of $Y$, see [13, Thm. 1.2b] or [7, Sect. 8.1]. In particular, the nonabelian Hodge correspondence for klt spaces, [9, Thm. 3.4], applies to yield a locally free Higgs sheaf $\left(\mathscr{F}_{Y}, \theta_{\mathscr{F}_{Y}}\right):=$ $\eta_{Y}\left(F_{Y}\right) \in \operatorname{Higgs}_{Y}$. More is true: We have seen in [9, Prop. 3.11] that $\left.\left(\mathscr{F}_{Y}, \theta_{\mathscr{F}_{Y}}\right)\right|_{Y^{\circ}}$ admits a tame, purely imaginary harmonic bundle structure $\mathbb{F}_{Y^{\circ}}^{\prime}$ whose associated local system $F_{Y^{\circ}}^{\prime}$ is isomorphic to $F_{Y^{\circ}}$. Corollary 3.14 thus yields the desired isomorphism (3.17.1).

Corollary 3.19 (Boundedness of TPI-Higgs) Let X be a projective klt space. Then, the families TPI-Higgs $_{X_{\mathrm{reg}}}$ and TPI-locFree $X_{\mathrm{reg}}$ are bounded, and so is the family

TPI-refl $X:=$ iso. classes of reflexive sheaves $\mathscr{F}$ on $X$ with $\left.\mathscr{F}\right|_{X_{\mathrm{reg}}} \in \mathrm{TPI}_{\mathrm{T}}$ locFree $X_{\mathrm{reg}_{\mathrm{reg}}}$.

Proof. Choose a maximally quasi-étale cover $\gamma: Y \rightarrow X$, Galois with group $G$, and use the same notation $X^{\circ}, Y^{\circ}$ and $\delta$ as in Proposition 3.17. Recall from [9, Cor. 7.2] that the family $\operatorname{Higgs}_{Y}$ is bounded. Standard arguments, which we leave to the reader, show that the following families are likewise bounded

$\mathbb{A}_{1}:=\left\{\right.$ loc. free Higgs $G$-sheaves on $Y$ whose underlying Higgs sheaf is in $\left.\operatorname{Higgs}_{Y}\right\}$

$\mathbb{A}_{2}:=\left\{\right.$ loc. free Higgs sheaves on $X^{\circ}$ of the form $\delta_{*}\left(\left.\left(\mathscr{F}_{Y}, \theta_{\mathscr{F}_{Y}}\right)\right|_{Y^{\circ}}\right)^{G}$,

$$
\left.\left(\mathscr{F}_{Y}, \theta_{\mathscr{F}_{Y}}\right) \text { in } \mathbb{A}_{1}\right\} \text {. }
$$

But we have seen in Proposition 3.17 and Remark 3.18 that every locally free Higgs sheaf in TPI-Higgs $X_{\text {reg }}$ appears in $\mathbb{A}_{2}$. This proves boundedness of TPI-Higgs $X_{\text {reg }}$ and TPI-locFree $X_{\text {reg }}$. Finally, by considering the push-forward from $X_{\text {reg }}$ to $X$ and by using reflexivity of its members we conclude that TPI-refl $X_{X}$ is also bounded.

The following corollary is now a direct consequence of Remark 3.4, of the boundedness result above, of Corollary 3.16, and of the iterated Bertini-type theorem for bounded families, [9, Prop. 7.3]. We emphasise that it contains a criterion for a reflexive Higgs sheaf to be locally free.

Corollary 3.20 (Restriction criterion for TPI-Higgs) Let $X$ be a projective klt space of dimension $n>2$, let $H \in \operatorname{Div}(X)$ be ample, and let $\left(\mathscr{E}_{X_{\mathrm{reg}}}, \theta_{\mathscr{E}_{X_{\mathrm{reg}}}}\right)$ be a reflexive Higgs

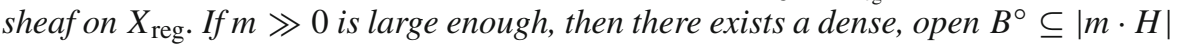
such that every hypersurface $D \in B^{\circ}$ is a klt space, satisfies $D_{\mathrm{reg}}=D \cap X_{\mathrm{reg}}$, and such that

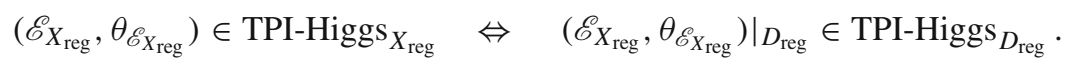

In plain words, the reflexive Higgs sheaf $\left(\mathscr{E}_{X_{\mathrm{reg}}}, \theta_{\mathscr{E}_{X_{\mathrm{reg}}}}\right)$ is locally free and admits a tame and purely imaginary harmonic bundle structure if and only if its restriction to $D_{\text {reg }}$ is locally free and admits a tame and purely imaginary harmonic bundle structure.

Proof The conditions on the singularities of the hypersurface $D$ stated above are met by a Zariski-open subset of any basepoint free linear system owing to Seidenberg's theorem [2, Thm. 1.7.1] and [20, Lem. 5.17]. 
The " $\Rightarrow$ " direction is contained in Remark 3.4. For the other direction, let $X^{\circ}$ be the big open subset of $X_{\text {reg }}$ consisting of points where $\mathscr{E}_{X_{\text {reg }}}$ is locally free, and let $F^{\circ}$ be the family of locally free Higgs sheaves $\left(\mathscr{F}_{X^{\circ}}, \theta_{\mathscr{F}_{X^{\circ}}}\right)$ obtained by restricting Higgs sheaves $\left(\mathscr{F}_{X_{\text {reg }}}, \theta_{\mathscr{F}_{X_{\text {reg }}}}\right) \in$ TPI-Higgs ${ }_{X_{\text {reg }}}$ to $X^{\circ}$. From Corollary 3.19 above we obtain that $F^{\circ}$ is bounded (as a family of Higgs sheaves). Let $m \gg 0$ be large enough and $D \in|m H|$ be general enough for both Corollary 3.16 and [9, Prop. 7.3] to apply to $D$ (as the first member of a tuple) with respect to $F^{\circ}$ and $\left(\mathscr{E}_{X^{\circ}}, \theta_{\mathscr{E}_{X^{\circ}}}\right):=\left.\left(\mathscr{E}_{X_{\text {reg }}}, \theta_{\mathscr{E}_{X_{\text {reg }}}}\right)\right|_{X^{\circ}}$. By assumption, $\left.\left(\mathscr{E}_{X_{\text {reg }}}, \theta_{\mathscr{E}_{X_{\text {reg }}}}\right)\right|_{D_{\text {reg }}} \in$ TPI-Higgs $D_{\text {reg }}$, and hence Corollary 3.16 implies that there exists a locally free Higgs sheaf $\left(\mathscr{F}_{X_{\text {reg }}}, \theta_{\mathscr{F}_{X_{\text {reg }}}}\right) \in$ TPI-Higgs $_{X_{\text {reg }}}$ whose restriction to $D_{\text {reg }}$ is isomorphic to $\left.\left(\mathscr{E}_{X_{\text {reg }}}, \theta_{\mathscr{E}_{X_{\text {reg }}}}\right)\right|_{D_{\text {reg }}}$. Choosing a smooth, projective curve $C \subset D \cap X^{\circ}$ as in [9, Prop. 7.3], it clearly follows that $\left.\left(\mathscr{F}_{X^{\circ}}, \theta_{\mathscr{F}_{X^{\circ}}}\right)\right|_{C} \cong$ $\left.\left(\mathscr{E}_{X^{\circ}}, \theta_{\mathscr{E}_{X^{\circ}}}\right)\right|_{C}$, from which we infer in a first step using loc. cit. that $\left(\mathscr{F}_{X^{\circ}}, \theta_{\mathscr{F}_{X^{\circ}}}\right) \cong$ $\left(\mathscr{E}_{X^{\circ}}, \theta_{\mathscr{E}_{X^{\circ}}}\right)$ and in a second step using reflexivity of both $\mathscr{F}_{X_{\text {reg }}}$ and $\mathscr{E}_{X_{\text {reg }}}$ that

$$
\left(\mathscr{E}_{X_{\text {reg }}}, \theta_{\mathscr{E}_{X_{\text {reg }}}}\right) \cong\left(\mathscr{F}_{X_{\text {reg }}}, \theta_{\mathscr{F}_{X_{\text {reg }}}}\right) \in \text { TPI-Higgs } X_{\text {reg }}
$$

\section{Higgs bundles and Higgs sheaves}

To prepare for the proof of the uniformisation result in the Part II of this paper and to fix notation, we briefly recall the notion of $\mathbb{Q}$-varieties in the specific case of surfaces; details concerning this notion can be found in [8, Part I]. Section 4.2 discusses stability notions for Higgs sheaves that are defined only on the smooth locus of a projective klt space.

\subsection{Higgs sheaves on $\mathbb{Q}$-surfaces}

For surfaces with quotient singularities, we show how a Higgs $\mathbb{Q}$-sheaf can be constructed from a Higgs sheaf that is defined on the smooth locus of the underlying surface; the theory can certainly be developed to cover more general cases, but we restrict ourselves to the material necessary for the arguments in the proof of our main result. For further material, the reader is referred to [8, Sect. 5.5], where the notion of Higgs $\mathbb{Q}$-sheaves is defined in general.

Construction 4.1 (Quasi-étale $\mathbb{Q}$-structure on a surface with quotient singularities) Let $S$ be a normal, projective surface with only quotient singularities. By [8, Prop. 3.10], the surface $S$ can then be equipped with the structure of a quasi-étale $\mathbb{Q}$-variety in the sense of [8, Defs. 3.1 and 3.2]. Choosing one such structure, say $S^{\mathbb{Q}}$, we are given a finite set $A$ and for each $\alpha \in A$ a smooth, quasi-projective variety $S_{\alpha}$ and a diagram

$$
S_{\alpha} \underset{\text { Galois with group } G_{\alpha}}{p_{\alpha}, \text { quasi-étale }}
$$


such that images $p\left(S_{\alpha}\right)$ cover $S$, and such that certain compatibility conditions hold; we refer the reader to [8, Sect. 3.3] for a further discussion.

Next, recall from Mumford in [30, Sect. 2] or [8, Sect. 3.4] that $S^{\mathbb{Q}}$ admits a global, Cohen-Macaulay cover. In other words, there exists a finite, Galois morphism $\gamma: \widehat{S} \rightarrow S$ from a normal (and hence Cohen-Macaulay) surface, with Galois group $G:=\operatorname{Gal}(\widehat{S} / S)$, and for every $\alpha \in A$ a commutative diagram as follows,

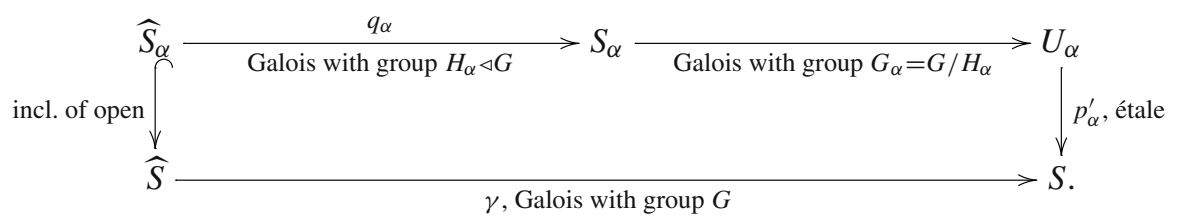

Construction 4.2 (Higgs $\mathbb{Q}$-bundle from Higgs bundle on $S_{\text {reg }}$ ). In the setup of Construction 4.1, assume that $S_{\text {reg }}$ is equipped with a locally free Higgs sheaf $\left(\mathscr{E}_{S_{\text {reg }}}, \theta_{\mathscr{E}_{S_{\text {reg }}}}\right)$. We will denote the reflexive extension of $\mathscr{E}_{S_{\text {reg }}}$ to $S$ by $\mathscr{E}_{S}$. Slightly generalising [8, Constructions 3.8 and 5.15], one constructs a locally free Higgs $\mathbb{Q}$-sheaf $\left(\mathscr{E}_{S}, \theta_{\mathscr{E}_{S}}\right)^{[\mathbb{Q}]}$ on $S^{\mathbb{Q}}$, given by the collection $\left(\mathscr{E}_{S_{\alpha}}, \theta_{\mathscr{E}_{S_{\alpha}}}\right)$ of locally free Higgs sheaves on the $S_{\alpha}$ that can be obtained by extending the Higgs bundles $\left(\left.p_{\alpha}\right|_{p_{\alpha}^{-1}\left(S_{\text {reg }}\right)}\right)^{*}\left(\mathscr{E}_{S_{\text {reg }}}, \theta_{\mathscr{E}_{\text {reg }}}\right)$ to a Higgs bundle on $S_{\alpha}$ using the Riemann Extension Theorem.

More precisely, we set $S_{\alpha}^{\circ}=S_{\alpha} \backslash p_{\alpha}^{-1}\left(S_{\text {sing }}\right)$. Each $\mathscr{E}_{S_{\alpha}}:=\left(\left.p_{\alpha}\right|_{p_{\alpha}^{-1}\left(S_{\text {reg }}\right)}\right)^{*}(\mathscr{E})$ extends to a locally free sheaf $\mathscr{E}_{S_{\alpha}}$ on $S_{\alpha}$. In addition, as the induced Higgs fields on $\mathscr{E}_{S_{\alpha}}$ are sections of $\mathscr{E} n d\left(\mathscr{E}_{S_{\alpha}}\right) \otimes \Omega_{S_{\alpha}^{\circ}}^{1}$, they extend to sections of $\mathscr{E} n d\left(\mathscr{E}_{S_{\alpha}}\right) \otimes \Omega_{S_{\alpha}}^{1}$. We denote these extended sections by $\theta_{\mathscr{E}_{S_{\alpha}}}$. Now, the pull-backs $q_{\alpha}^{*}\left(\mathscr{E}_{S_{\alpha}}, \theta_{\mathscr{E}_{S_{\alpha}}}\right)$ are locally free Higgs sheaves on $\widehat{S}_{\alpha}$ that glue to give a locally free Higgs $G$-sheaf $\left(\mathscr{E}_{\widehat{S}}, \theta_{\mathscr{E}_{\widehat{S}}}\right)$ on $\widehat{S}$. The construction works without change for sheaves without (or with the trivial) Higgs field.

Remark 4.3 In Construction 4.2, we have $\mathscr{E}_{\widehat{S}} \cong \gamma^{[*]} \mathscr{E}_{S}$ and therefore $\gamma_{*}\left(\mathscr{E}_{\widehat{S}}\right)^{G} \cong \mathscr{E}_{S}$.

Construction 4.4 (Pull-back out of a $\mathbb{Q}$-structure) In the setting of Construction 4.2, consider a $G$-equivariant resolution of singularities, $\pi: \widetilde{S} \rightarrow \widehat{S}$. Given an arbitrary Higgs sheaf $(\mathscr{F}, \tau)$ on $\widehat{S}$, there is generally no way to define a Higgs field on the pull-back $\pi^{*} \mathscr{F}$ even in cases where $\mathscr{F}$ is locally free. In our special setting, however, recall from [8, Lem. 5.17] that there exists a $G$-invariant Higgs field on the $G$-sheaf $\mathscr{E}_{\widetilde{S}}:=\pi^{*} \mathscr{E}_{\widehat{S}}$ that agrees with the pull-back of $\theta_{\mathscr{E}_{\widehat{S}}}$ wherever $\widehat{S}$ is smooth.

\subsection{Stability and polystability}

Stability properties of Higgs sheaves on singular, projective varieties are defined and discussed in detail in [8, Sect. 5.6]. In the situation at hand, it makes sense to generalise this to the case where a Higgs sheaf is defined on the smooth locus only. We recall [9, Def. 2.19] in our setting.

Definition 4.5 (Stability for Higgs sheaves on the smooth locus) Let $X$ be a normal, projective variety, let $H \in \operatorname{Div}(X)$ be nef and let $\left(\mathscr{E}_{X_{\text {reg }}}, \theta_{\mathscr{E}_{X_{\text {reg }}}}\right)$ be a torsion free Higgs 
sheaf on $X_{\text {reg }}$. We say that $\left(\mathscr{E}_{X_{\text {reg }}}, \theta_{\mathscr{E}_{X_{\text {reg }}}}\right)$ is stable with respect to $H$ if any generically Higgs-invariant subsheaf $\mathscr{F}_{X_{\text {reg }}} \subsetneq \mathscr{E}_{X_{\text {reg }}}$ with $0<$ rank $\mathscr{F}_{X_{\text {reg }}}<$ rank $\mathscr{E}_{X_{\text {reg }}}$ satisfies

$$
\frac{c_{1}\left(\iota_{*} \mathscr{F}_{X_{\mathrm{reg}}}\right) \cdot[H]}{\operatorname{rank} \mathscr{F}_{X_{\mathrm{reg}}}}<\frac{c_{1}\left(\iota_{*} \mathscr{E}_{X_{\mathrm{reg}}}\right) \cdot[H]}{\operatorname{rank} \mathscr{E}_{X_{\mathrm{reg}}}}
$$

where $\iota: X_{\text {reg }} \rightarrow X$ is the inclusion. Analogously, we define semistable and polystable.

The stability notion of Definition 4.5 is compatible with the existing notions. The proof of the following fact is elementary and therefore omitted.

Fact 4.6 (Compatibility with existing notions, [9, Lem. 2.21]). Let X be a normal, projective variety, let $H \in \operatorname{Div}(X)$ be nef, and let $\left(\mathscr{E}_{X}, \theta_{\mathscr{E}_{X}}\right)$ be a reflexive Higgs sheaf that is defined on all of $X$. Then, the following are equivalent.

(4.6.1) The sheaf $\left(\mathscr{E}_{X}, \theta_{\mathscr{E}_{X}}\right)$ is stable with respect to $H$.

(4.6.2) The sheaf $\left.\left(\mathscr{E}_{X}, \theta_{\mathscr{E}_{X}}\right)\right|_{X_{\text {reg }}}$ is stable with respect to $H$.

The analogous statements hold in the semistable and polystable setting.

The following lemma discusses behaviour of stability when taking tensor products, and shows polystability for Higgs bundles in TPI-Higgs.

Lemma 4.7 (Polystability in tensor products and TPI-Higgs). Let $X$ be a normal, projective variety, let $H$ be an ample divisor on $X$, and let $\left(\mathscr{F}_{X_{\mathrm{reg}}}, \theta_{\mathscr{F}_{X_{\mathrm{reg}}}}\right)$ be a Higgs

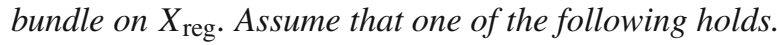

(4.7.1) The Higgs bundle $\left(\mathscr{F}_{X_{\text {reg }}}, \theta_{\mathscr{F}_{X_{\text {reg }}}}\right)$ is in TPI-Higgs $X_{\text {reg }}$.

(4.7.2) The Higgs bundle $\left(\mathscr{F}_{X_{\mathrm{reg}}}, \theta_{\mathscr{F}_{\mathrm{reg}}}\right)$ is a tensor product of two Higgs bundles on $X_{\text {reg }}$ that are stable with respect to $H$.

Then, $\left(\mathscr{F}_{X_{\mathrm{reg}}}, \theta_{\mathscr{F}_{X_{\mathrm{reg}}}}\right)$ is polystable with respect to $H$. In particular, if $\left(\mathscr{E}_{X_{\mathrm{reg}}}, \theta_{\mathscr{E}_{X_{\mathrm{reg}}}}\right)$ is a Higgs bundle on $X_{\mathrm{reg}}$ that is stable with respect to $H$, then $\mathscr{E} n d\left(\mathscr{E}_{X_{\mathrm{reg}}}, \theta_{\mathscr{E}_{X_{\mathrm{reg}}}}\right)$ is polystable with respect to $H$.

Proof. To begin, we remark that $\left(\mathscr{F}_{X_{\text {reg }}}, \theta_{\mathscr{F}_{X_{\text {reg }}}}\right)$ is semistable. In fact, Remark 3.4 and the restriction theorem for semistable Higgs sheaves on $X_{\text {reg }}$, [9, Thm. 6.1], respectively, allows us to restrict ourselves to the case where $X=X_{\text {reg }}$ is a smooth projective curve. There, the result is classically known in either of the two cases.

The proof of polystability proceeds by induction on the dimension on $X$. If $\operatorname{dim} X=$ 1 , then $X$ is a smooth projective curve and the result is classically known in either case. As for the inductive step, assume that the result was known for all varieties of dimension less than $\operatorname{dim} X$, and assume that there exists a saturated, Higgs-invariant subsheaf $\mathscr{A}_{X_{\text {reg }}} \subsetneq \mathscr{F}_{X_{\text {reg }}}$ whose slope equals that of $\mathscr{F}_{X_{\text {reg }}}$ and that is stable with respect to $H$. We need to show that $\mathscr{A}_{X_{\text {reg }}}$ is a direct summand. More precisely, we need to find a morphism of Higgs sheaves, $\mathscr{F}_{X_{\text {reg }}} \rightarrow \mathscr{A}_{X_{\text {reg }}}$, that is a projection onto $\mathscr{A}_{X_{\text {reg }}}$.

Now, if $m \gg 0$ is sufficiently large and $Y \in|m \cdot H|$ is sufficiently general, then $Y$ is normal, $Y_{\text {reg }}=Y \cap X_{\text {reg }}$ and the following will hold. 
(4.7.3) The subsheaf $\left.\mathscr{A}_{X_{\text {reg }}}\right|_{\text {reg }} \subsetneq \mathscr{F} X_{\text {reg }} \mid Y_{\text {reg }}$ Higgs-invariant and stable. This is the Restriction Theorem for stable Higgs sheaves on $X_{\text {reg, }}$ [9, Thm. 6.1].

(4.7.4) The restriction $\operatorname{Hom}\left(\mathscr{F}_{X_{\text {reg }}}, \mathscr{A}_{X_{\text {reg }}}\right) \rightarrow \operatorname{Hom}\left(\left.\mathscr{F}_{X_{\text {reg }}}\right|_{Y_{\text {reg }}},\left.\mathscr{A}_{X_{\text {reg }}}\right|_{Y_{\text {reg }}}\right)$ is bijective. This follows from standard arguments, compare [9, Prop. 7.3 and proof].

Item (4.7.3) together with the induction hypothesis show that there exists a projection map $\left.\left.\mathscr{F}_{X_{\text {reg }}}\right|_{Y_{\text {reg }}} \rightarrow \mathscr{A}_{X_{\text {reg }}}\right|_{Y_{\text {reg }}}$ respecting Higgs fields. Item (4.7.4) implies that this map extends to a projection $\mathscr{F}_{X_{\text {reg }}} \rightarrow \mathscr{A}_{X_{\text {reg }}}$, presenting $\mathscr{A}_{X_{\text {reg }}}$ as a direct summand of $\mathscr{F}_{X_{\text {reg }}}$. A variant of (4.7.4) that we leave to the reader, using morphisms of Higgssheaves instead of sheaf morphisms, shows that for $m$ sufficiently large, the projection $\mathscr{F}_{X_{\text {reg }}} \rightarrow \mathscr{A}_{X_{\text {reg }}}$ is in fact a morphism of Higgs sheaves, cf. [22, Lem. 5] and [25, Lem. 3.4].

\section{Existence of harmonic structures}

The following is the main result in Part I of the present paper. It generalises earlier results obtained in $[7,23,28,34]$.

Theorem 5.1 (Existence of harmonic structures) Let $X$ be a projective klt space of dimension $n \geq 2$. Let $H \in \operatorname{Div}(X)$ be ample and use $H$ to equip $X_{\text {reg }}$ with a Kähler metric. Let $\left(\mathscr{E}_{X_{\mathrm{reg}}}, \theta_{\mathscr{E}_{\mathrm{X}} \mathrm{reg}}\right)$ be a reflexive Higgs sheaf on $X_{\mathrm{reg}}$. Denote the reflexive extension of $\mathscr{E}_{X_{\mathrm{reg}}}$ to $X$ by $\mathscr{E}_{X}$. Then, the following statements are equivalent.

(5.1.1) The Higgs sheaf $\left(\mathscr{E}_{X_{\mathrm{reg}}}, \theta_{\mathscr{E}_{X_{\mathrm{reg}}}}\right)$ is (poly)stable with respect to $H$ and the $\mathbb{Q}$ Chern characters satisfy

$$
\widehat{c h}_{1}\left(\mathscr{E}_{X}\right) \cdot[H]^{n-1}=0 \text { and } \widehat{c h}_{2}\left(\mathscr{E}_{X}\right) \cdot[H]^{n-2}=0 .
$$

(5.1.2) The sheaf $\mathscr{E}_{X_{\mathrm{reg}}}$ is locally free and $\left(\mathscr{E}_{X_{\mathrm{reg}}}, \theta_{\mathscr{E}_{X_{\mathrm{reg}}}}\right)$ is induced by a tame, purely imaginary harmonic bundle whose associated flat bundle is (semi)simple.

\subsection{Proof of Theorem 5.1}

The two implications are proven separately.

Implication $(5.1 .1) \Rightarrow(5.1 .2)$

Suppose we already know that $\left(\mathscr{E}_{X_{\text {reg }}}, \theta_{\mathscr{E}_{X \text { reg }}}\right)$ admits a tame and purely imaginary harmonic bundle structure $\mathbb{E}_{X_{\text {reg }}}$. Then the induced flat bundle $\left(E_{X_{\text {reg }}}, \nabla_{\mathbb{E}_{X_{\text {reg }}}}\right)$ is always semisimple, see Remark 3.10. If $\left(\mathscr{E}_{X_{\text {reg }}}, \theta_{\mathscr{E}_{X_{\text {reg }}}}\right)$ is even stable, then Lemma 3.12 implies that $\left(E_{X_{\text {reg }}}, \nabla_{\mathbb{E}_{X_{\text {reg }}}}\right)$ is simple. It therefore remains to establish a tame, purely imaginary harmonic bundle structure. The proof is rather long, and therefore subdivided into six steps. 


\section{Implication $(5.1 .1) \Rightarrow(5.1 .2)$, Step 1: Reduction to stable sheaves on surfaces}

Standard arguments involving the Bogomolov-Gieseker inequality show that it suffices to consider the stable case only. We refer to [6, Step 2 in proof of Thm. 6.2] for details.

Assumption w.l.o.g. 5.2 The Higgs sheaf $\left(\mathscr{E}_{X_{\text {reg }}}, \theta_{\mathscr{E}_{X_{\text {reg }}}}\right)$ is stable with respect to $H$.

Choosing a sufficiently increasing sequence of numbers $0 \ll m_{1} \ll m_{2} \ll \cdots \ll$ $m_{n-2}$ as well as a sufficiently general tuple of hyperplanes,

$$
\left(D_{1}, \ldots, D_{n-2}\right) \in\left|m_{1} \cdot H\right| \times \cdots \times\left|m_{n-2} \cdot H\right| \text {, }
$$

the following will hold.

(5.3.1) The intersection $S:=D_{1} \cap \cdots \cap D_{n-2}$ is irreducible and normal. Moreover, $S$

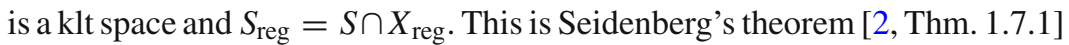
and [20, Lem. 5.17].

(5.3.2) The restricted sheaf $\mathscr{E}_{S}:=\left.\mathscr{E}_{X}\right|_{S}$ is reflexive, hence locally free on $S_{\text {reg }}$, and satisfies $\widehat{c h}_{2}\left(\mathscr{E}_{S}\right)=0$ as well as $\widehat{c}_{1}\left(\mathscr{E}_{Y}\right) \cdot\left[\left.H\right|_{S}\right]=0$. This is [16, Cor. 1.1.14] and [8, Thm. 3.13].

(5.3.3) The locally free Higgs sheaf $\left(\mathscr{E}_{S_{\text {reg }}}, \theta_{\mathscr{E}_{\text {reg }}}\right):=\left.\left(\mathscr{E}_{X_{\text {reg }}}, \theta_{\mathscr{E}_{X_{\text {reg }}}}\right)\right|_{S_{\text {reg }}}$ is stable with respect to $\left.H\right|_{S}$. This is the Restriction Theorem [9, Thm. 6.1].

(5.3.4) The reflexive Higgs sheaf $\left(\mathscr{E}_{X_{\text {reg }}}, \theta_{\mathscr{E}_{X_{\text {reg }}}}\right)$ is locally free and admits a tame and purely imaginary harmonic bundle structure if and only if the locally free Higgs sheaf $\left.\left(\mathscr{E}_{X_{\text {reg }}}, \theta_{\mathscr{E}_{X_{\text {reg }}}}\right)\right|_{S_{\text {reg }}}$ admits a tame and purely imaginary harmonic bundle structure. This is Corollary 3.20.

Items (5.3.1)-(5.3.3) imply that the surface $S$ and the Higgs sheaf $\left.\left(\mathscr{E}_{X_{\text {reg }}}, \theta_{\mathscr{E}_{X_{\text {reg }}}}\right)\right|_{S_{\text {reg }}}$ reproduce the assumptions made in Theorem 5.1. Item (5.3.4) implies that it suffices to show that the locally free Higgs sheaf $\left.\left(\mathscr{E}_{X_{\text {reg }}}, \theta_{\mathscr{E}_{X_{\text {reg }}}}\right)\right|_{S_{\text {reg }}}$ admits a tame and purely imaginary harmonic bundle structure. Replacing $X$ by $S$ we can (and will) therefore assume the following.

Assumption w.l.o.g.5.3 The dimension of $X$ is equal to two.

\section{Implication $(5.1 .1) \Rightarrow(5.1 .2)$, Step 2: The $\mathbb{Q}$-structure}

As we have seen in (5.3.2) above, assumption 5.3 immediately implies that the reflexive sheaf $\mathscr{E}_{X_{\text {reg }}}$ is locally free. In a similar vein, since $X$ is a klt surface, we obtain immediately that $X$ has quotient singularities. We may therefore equip $X$ with a quasiétale $\mathbb{Q}$-structure and choose a global Cohen-Macaulay cover with Galois group $G$ allowing to compute $\mathbb{Q}$-Chern classes as in [8, Thm. 3.13], and a $G$-equivariant, strong $\log$-resolution of singularities as follows,

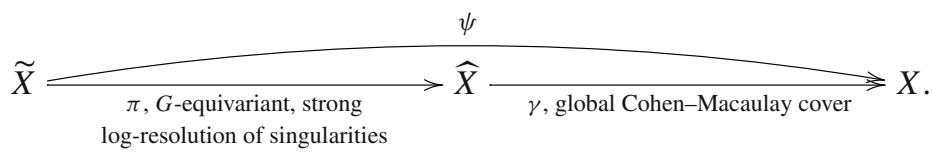


The locally free Higgs $G$-bundle on $\widetilde{X}$ obtained in Construction 4.4 will be denoted by $\left(\mathscr{E} \widetilde{X}, \theta_{\mathscr{E}}\right)$. The following immediate consequence of Assumption 5.2 will be used later.

Claim 5.4 (Stability and Chern classes of $\left.\mathscr{E}_{\widetilde{X}}\right)$ The Higgs $G$-bundle $\left(\mathscr{E}_{\widetilde{X}}, \theta_{\mathscr{E} \widetilde{X}}\right)$ is $G$ stable with respect to a $G$-invariant ample divisor $\widetilde{H} \in \operatorname{Div}(\widetilde{X})$. The Chern classes $c_{i}\left(\mathscr{E}_{\widetilde{X}}\right) \in H^{2 i}(\widetilde{X}, \mathbb{R})$ vanish.

Proof of Claim 5.4 The first claim is proven as in the proof of [8, Prop. 6.2], using the locally free Higgs $G$-sheaf $\left(\mathscr{E}_{\widehat{X}}, \theta_{\mathscr{E}_{\widehat{X}}}\right)$ on $\widehat{X}$ obtained in Construction 4.2. For the second claim, observe that the maximally destabilising subsheaf of the Higgs bundle ${ }^{3}$ $\left(\mathscr{E}_{\widetilde{X}}, \theta_{\mathscr{E}_{\tilde{X}}}\right)$ with respect to $\widetilde{H}$ is automatically $G$-invariant. In particular, it follows from $G$-stability that $\left(\mathscr{E}_{\widetilde{X}}, \theta_{\mathscr{E}_{\tilde{X}}}\right)$ is at least semistable with respect to $\widetilde{H}$. Moreover, from (5.3.2), from [8, Thm. 3.13], and from the functorial properties of Chern classes $\left[4\right.$, Thm. 3.2(d)] we conclude that $c_{1}\left(\mathscr{E}_{\tilde{X}}\right) \cdot \widetilde{H}=c h_{2}\left(\mathscr{E}_{\tilde{X}}\right)=0$. Vanishing of the $c_{i}$ hence follows from the Hodge index theorem and the Bogomolov-Gieseker inequality as in the first paragraph of [6, Sect. 6.3].

$\square$ (Claim 5.4)

For the remainder of the proof, we fix one $G$-invariant ample divisor $\widetilde{H} \in \operatorname{Div}(\tilde{X})$ as in Claim 5.4 and use this divisor to equip $\widetilde{X}$ with a $G$-invariant Kähler metric $\omega_{\tilde{H}}$. We denote the holomorphic vector bundle associated with $\mathscr{E}_{X_{\mathrm{reg}}}$ by $\left(E_{X_{\mathrm{reg}}}, \bar{\partial}_{E_{X_{\mathrm{reg}}}}\right)$. We use similar notation also for other bundles, including $\left(E_{\widetilde{X}}, \bar{\partial}_{E_{\tilde{X}}}\right)$.

\section{Open sets}

Throughout the proof, we consider the big, open subset $X^{\circ}:=X_{\text {reg }} \backslash \gamma\left(\widehat{X}_{\text {sing }}\right)$ of $X$. Preimages and restricted morphisms are written as

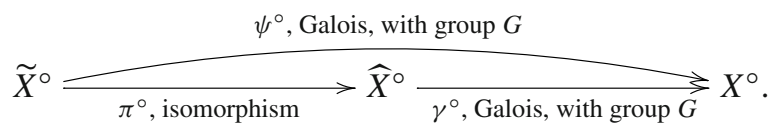

Write $\left(\mathscr{E}_{X^{\circ}}, \theta_{X^{\circ}}\right):=\left.\left(\mathscr{E}_{X_{\text {reg }}}, \theta_{X_{\text {reg }}}\right)\right|_{X^{\circ}}$. We use similar notation also for other open sets and write $\left(\mathscr{E}_{\tilde{X}^{\circ}}, \theta_{\mathscr{E}_{\tilde{X}^{\circ}}}\right)$.

\section{Orbifold charts}

We also consider orbifold charts, as introduced in Construction 4.1. The relevant morphisms and their restrictions are summarised in the following diagram,

\footnotetext{
$\overline{{ }^{3} \text { Here, we view }\left(\mathscr{E}_{\tilde{X}}, \theta_{\mathscr{E}} \widetilde{X}\right.}$ as a Higgs-bundle without its structure as a $G$-sheaf 


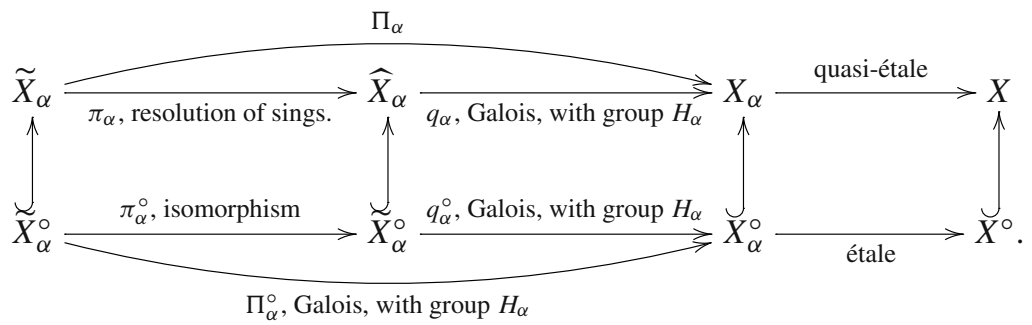

\section{Implication (5.1.1) $\Rightarrow$ (5.1.2), Step 3: Simplification}

The following claim allows us to concentrate on the big, open set $X^{\circ} \subseteq X_{\text {reg, simpli- }}$ fying notation substantially.

Claim 5.5 To prove Theorem 5.1, it suffices to show that the Higgs bundle $\left(\mathscr{E}_{X^{\circ}}, \theta_{\mathscr{E}_{X^{\circ}}}\right)$ admits a tame and purely imaginary harmonic bundle structure.

Proof of Claim 5.5 This is a direct consequence of Fact 2.6 and Corollary 3.14. A detailed proof is found in the arXiv version of this paper.

$\square$ (Claim 5.5)

\section{Implication (5.1.1) $\Rightarrow$ (5.1.2), Step 4: G-invariant harmonic structure on $\boldsymbol{E}_{\tilde{X}}$}

To start the core of the argument, we will now show the existence of a $G$-invariant harmonic structure on $\left(\mathscr{E}_{\widetilde{X}}, \theta_{\mathscr{E}_{\widetilde{X}}}\right)$.

Claim 5.6 The locally free Higgs $G$-sheaf $\left(\mathscr{E}_{\widetilde{X}}, \theta_{\mathscr{E}_{\tilde{X}}}\right)$ admits a $G$-invariant harmonic bundle structure $\mathbb{E}_{\tilde{X}}:=\left(E_{\widetilde{X}}, \bar{\partial}_{E_{\tilde{X}}}, \theta_{\mathscr{E}_{\tilde{X}}}, h_{E_{\widetilde{X}}}\right)$.

Proof of Claim 5.6 The $G$-stability found in Claim 5.4 allows us to apply a classical result of Simpson, [36, Thm. 1 on p. 878], which states that that $E_{\widetilde{X}}$ carries a $G$ invariant Hermitian metric $h_{E_{\widetilde{X}}}$ that is Hermitian Yang-Mills with respect to the Kähler metric $\omega_{\widetilde{H}}$. Using the notation of Fact and Definition 3.1, vanishing of Chern classes, Claim 5.4, and [37, discussion on p. 16f] imply that the connection

$$
\nabla_{\mathbb{E}_{\tilde{X}}}:=\partial_{E_{\widetilde{X}}}+\bar{\partial}_{E_{\widetilde{X}}}+\theta_{\mathscr{E}_{\widetilde{X}}}+\theta_{\mathscr{E}_{\tilde{X}}}^{h_{\tilde{X}}}
$$

is in fact flat and hence that $\left(E_{\widetilde{X}}, \bar{\partial}_{E_{\tilde{X}}}, \theta_{\mathscr{E}_{\tilde{X}}}, h_{E_{\tilde{X}}}\right)$ is a harmonic bundle.

$\square($ Claim 5.6)

Claim 5.7 If $F_{\widetilde{X}}$ is a $G$-invariant flat subbundle of $E_{\widetilde{X}}$, then either $F_{\widetilde{X}}=0$ or $F_{\widetilde{X}}=E_{\widetilde{X}}$.

Proof of Claim 5.7 We have seen in Lemma 3.12 that $F_{\widetilde{X}}$ is a holomorphic subbundle of $\left(E_{\widetilde{X}}, \bar{\partial}_{E_{\tilde{X}}}\right)$ and yields a Higgs-invariant, locally free subsheaf $\mathscr{F}_{\widetilde{X}}$ of $\left(\mathscr{E}_{\widetilde{X}}, \theta_{\mathscr{E}_{\tilde{X}}}\right)$. The assumption that $F_{\widetilde{X}}$ is a $G$-invariant subbundle of $E_{\tilde{X}}$ guarantees that $\mathscr{F}_{\tilde{X}}$ is a Higgsinvariant $G$-subsheaf of the Higgs $G$-sheaf $\left(\mathscr{E}_{\widetilde{X}}, \theta_{\mathscr{E}_{\tilde{X}}}\right)$. Using that $F_{\widetilde{X}}$ is invariant with respect to the flat connection $\nabla_{\mathbb{E}_{\tilde{X}}}$, we obtain that $F_{\widetilde{X}}$ is again flat, so that all its Chern classes vanish. Claim 5.7 thus follows from Claim 5.4 above.

$\square$ (Claim 5.7) 
Claim 5.8 If $F_{\widetilde{X}^{\circ}}$ is a $G$-invariant flat subbundle of $\left(E_{\widetilde{X}^{\circ}}, \nabla_{\mathbb{E}_{\tilde{X}}} \mid \widetilde{X}^{\circ}\right)$, then $F_{\widetilde{X}^{\circ}}$ extends to a $G$-invariant flat subbundle of $E_{\tilde{X}}$.

Proof of Claim 5.8 The question is local over the $X_{\alpha}$. More precisely, using the notation introduced in Step 2, it suffices to show that for every index $\alpha$, the restricted bundle $F_{\widetilde{X}_{\alpha}^{\circ}}:=\left.F_{\widetilde{X}^{\circ}}\right|_{\widetilde{X}_{\alpha}^{\circ}}$ on $\widetilde{X}_{\alpha}^{\circ}$ extends to a subbundle $F_{\widetilde{X}_{\alpha}} \subseteq E_{\widetilde{X}_{\alpha}}$ on $\widetilde{X}_{\alpha}$ that is invariant with respect to $\nabla_{\mathbb{E}_{\tilde{X}}}$. The $G$-invariance follows then automatically from density of $\widetilde{X}_{\alpha}^{\circ} \subseteq \widetilde{X}_{\alpha}$.

Recall from Constructions 4.2 and 4.4 that $E_{\widetilde{X}_{\alpha}^{\circ}}$ is a pull-back from $X_{\alpha}^{\circ}$, say $E_{\widetilde{X}_{\alpha}^{\circ}} \cong$ $\left(\Pi_{\alpha}^{\circ}\right)^{*} E_{X_{\alpha}^{\circ}}$. We claim that both the connection $\left.\nabla_{\mathbb{E}_{\tilde{X}}}\right|_{\widetilde{X}^{\circ}}$ and the subbundle $F_{\widetilde{X}_{\alpha}^{\circ}}$ descend to $X_{\alpha}^{\circ}$, too. Indeed, since $\nabla_{\mathbb{E}_{\tilde{X}}}$ is invariant under $G$, and hence also invariant under the Galois group $H_{\alpha}=\operatorname{Gal}\left(\Pi_{\alpha}^{\circ}\right) \subseteq G$, we may apply Proposition 2.7 to show the existence of a connection $\nabla_{E_{X_{\alpha}^{\circ}}}$ on $E_{X_{\alpha}^{\circ}}$ such that $\left.\nabla_{\mathbb{E}_{\tilde{X}}}\right|_{\widetilde{X}^{\circ}}=\left(\Pi_{\alpha}^{\circ}\right)^{*} \nabla_{E_{X_{\alpha}^{\circ}}}$. Moreover, it follows from [8, Prop. 2.16] applied to the associated locally free sheaves of $\mathscr{O} \tilde{X}_{\alpha}^{\circ}$-modules that there exists a subbundle $F_{X_{\alpha}^{\circ}} \subseteq E_{X_{\alpha}^{\circ}}$ with $F_{\widetilde{X}_{\alpha}^{\circ}}=\left(\Pi_{\alpha}^{\circ}\right)^{*} F_{X_{\alpha}^{\circ}}$. The subbundle $F_{X_{\alpha}^{\circ}}$ is then clearly invariant with respect to the connection $\nabla_{E_{X_{\alpha}^{\circ}}}$.

As $X_{\alpha}^{\circ}$ is a big open subset of the smooth, quasi-projective surface $X_{\alpha}$, the natural morphism of fundamental groups, $\pi_{1}\left(X_{\alpha}^{\circ}\right) \rightarrow \pi_{1}\left(X_{\alpha}\right)$, is isomorphic. Fact 2.6 therefore asserts that the subbundle $F_{X_{\alpha}^{\circ}} \subseteq E_{X_{\alpha}^{\circ}}$ extends from $X_{\alpha}^{\circ}$ to a $\nabla_{E_{X_{\alpha}^{\circ}}}$-invariant subbundle $F_{X_{\alpha}^{\circ}} \subseteq E_{X_{\alpha}^{\circ}}$ that exists on all of $X_{\alpha}$. Pulling back, we define the desired extension of $F_{\widetilde{X}_{\alpha}^{\circ}}$ as $F_{\widetilde{X}_{\alpha}}:=\left(\Pi_{\alpha}^{\circ}\right)^{*} F_{X_{\alpha}^{\circ}}$.

$\square$ (Claim 5.8)

Combining Claims 5.7 and 5.8, we arrive at the following result.

Construction 5.9 If $F_{\widetilde{X}^{\circ}}$ is a $G$-invariant subbundle of $E_{\widetilde{X}^{\circ}}$ that is also invariant with respect to $\left.\nabla_{\mathbb{E}_{\tilde{X}}}\right|_{\tilde{X}^{\circ}}$, then $F_{\widetilde{X}^{\circ}}=0$ or $F_{\widetilde{X}^{\circ}}=E_{\tilde{X}^{\circ}}$.

$\square$ (Consequence 5.9)

\section{Implication $(5.1 .1) \Rightarrow(5.1 .2)$, Step 5: Harmonic structure on $E_{X^{\circ}}$}

Eventually, we would like to show that the metric $h_{E_{\tilde{X}}}$ descends to a smooth Hermitian metric on $E_{X^{\circ}}$. However, owing to branching of the map $\gamma$ over the smooth part of $X_{\text {reg }}$, it is not clear from the outset whether the natural stratified $\mathcal{C}^{\infty}$-structure on the quotient $\tilde{X}^{\circ} / G$ will coincide with the $\mathcal{C}^{\infty}$-structure induced by the complex structure on $X^{\circ}$. Rather than showing descent of the metric directly, we will first discuss the flat structure on $E^{\circ}$ and construct a metric from there.

Write $\nabla_{E_{X^{\circ}}}$ for the unique connection on $E_{X^{\circ}}$ such that

$$
\left.\nabla_{\mathbb{E}_{\tilde{X}}}\right|_{\tilde{X}^{\circ}}=\left(\psi^{\circ}\right)^{*} \nabla_{E_{X^{\circ}}}
$$

which exists by Proposition 2.7. Consequence 5.9 implies that the flat bundle $\left(E_{X^{\circ}}, \nabla_{E_{X^{\circ}}}\right)$ is simple. We may hence apply the Jost-Zuo existence result for harmonic structures, Theorem 3.13 , to $\left(E_{X^{\circ}}, \nabla_{E_{X^{\circ}}}\right)$ and find a tame and purely imaginary, harmonic bundle structure $\mathbb{E}_{X^{\circ}}^{\prime}=\left(E_{X^{\circ}}, \bar{\partial}_{E_{X^{\circ}}}^{\prime}, \theta_{\mathscr{E}_{X^{\circ}}}^{\prime}, h_{E_{X^{\circ}}}^{\prime}\right)$ on $E_{X^{\circ}}$ whose associated connection $\nabla_{\mathbb{E}_{X^{\circ}}^{\prime}}$ equals $\nabla_{E_{X^{\circ}}}$. 


\section{Implication (5.1.1) $\Rightarrow$ (5.1.2), Step 6: Comparison}

There are now two tame and purely imaginary, $G$-invariant harmonic bundle structures on $E_{\widetilde{X}^{\circ}}$. First, the restriction $\left.\mathbb{E}_{\widetilde{X}}\right|_{\widetilde{X}^{\circ}}$, which is obviously tame and purely imaginary. The associated connection $\nabla_{\mathbb{E}_{\tilde{X}}} \widetilde{X}^{\circ}$ on $E_{\widetilde{X}^{\circ}}$ is semisimple by Remark 3.10. Second, by [27, Lem. 25.29] the pull-back of the harmonic structure $\left(\psi^{\circ}\right)^{*} \mathbb{E}_{X^{\circ}}^{\prime}$ on $E_{X^{\circ}}$ is also tame and purely imaginary. The associated connection on $E_{\tilde{X}^{\circ}}$ is the pull-back of $\nabla_{E_{X^{\circ}}}$, and hence likewise equal to $\nabla_{\mathbb{E}_{\tilde{X}}} \mid \widetilde{X}^{\circ}$.

Using the observation that the two tame and purely imaginary bundles induce the same semisimple connection, Theorem 3.13 now immediately implies that the complex structures and Higgs fields on $\widetilde{X}^{\circ}$ are equal

$$
\begin{aligned}
\left(\psi^{\circ}\right)^{*} \bar{\partial}_{E_{X^{\circ}}}=\left.\bar{\partial}_{E_{\tilde{X}}}\right|_{\widetilde{X}^{\circ}}=\left(\psi^{\circ}\right)^{*} \bar{\partial}_{E_{X^{\circ}}}^{\prime} \\
\left(\psi^{\circ}\right)^{*} \theta_{\mathscr{E}_{X^{\circ}}}=\left.\theta_{\mathscr{E}_{\tilde{X}}}\right|_{\widetilde{X}^{\circ}}=\left(\psi^{\circ}\right)^{*} \theta_{\mathscr{E}_{X^{\circ}}}^{\prime} .
\end{aligned}
$$

We emphasise that (5.10.1) is a statement about equalities of operators, rather than mere isomorphisms of complex structures and Higgs bundles. In particular, (5.10.1) implies that the holomorphic structure $\bar{\partial}_{E_{X^{\circ}}}$ corresponding to $\mathscr{E}_{X^{\circ}}$ equals $\bar{\partial}_{E_{X^{\circ}}}^{\prime}$ and furthermore that $\theta_{\mathscr{E}_{X^{\circ}}}=\theta_{\mathscr{E}_{X}}^{\prime}$ on $X^{\circ}$. Consequently, $\left(\mathscr{E}_{X_{\text {reg }}}, \theta_{\mathscr{E}_{X_{\text {reg }}}}\right) \in$ TPI-Higgs $X_{\text {reg }}$. The implication (5.1.1) $\Rightarrow$ (5.1.2) of Theorem 5.1 is thus established.

Implication $(5.1 .2) \Rightarrow(5.1 .1)$

First, remark that in the semisimple case the implication (5.1.2) $\Rightarrow(5.1 .1)$ is immediate consequence of Lemma 4.7, Proposition 3.17, [9, Thm. 3.10], and standard calculus of $\mathbb{Q}$-Chern classes; this is explained in detail in [8, Sect. 3.8].

Second, assume in addition that $\left(\mathscr{E}_{X_{\text {reg }}}, \theta_{\mathscr{E}_{\text {reg }}}\right) \in$ TPI-Higgs $X_{\text {reg }}$ is induced by a harmonic bundle $\mathbb{E}=\left(E, \bar{\partial}_{X_{\mathrm{reg}}}, \theta_{\mathscr{E}_{\mathrm{X}} \mathrm{reg}}, h\right)$ whose associated flat bundle $\left(E, \nabla_{\mathbb{E}}\right)$ is simple, but suppose that $\left(\mathscr{E}_{X_{\mathrm{reg}}}, \theta_{\mathscr{E}_{\mathrm{reg}}}\right)$ is not stable. Being polystable, it splits as a direct sum of stable bundles

$$
\left(\mathscr{E}_{X_{\mathrm{reg}}}, \theta_{\mathscr{E}_{\mathrm{X}} \mathrm{reg}}\right)=\left(\mathscr{E}_{X_{\mathrm{reg}}}^{(1)}, \theta_{\mathscr{E}_{X_{\mathrm{reg}}}}^{(1)}\right) \oplus \cdots \oplus\left(\mathscr{E}_{X_{\mathrm{reg}}}^{(m)}, \theta_{\mathscr{E}_{X_{\mathrm{reg}}}}^{(m)}\right) \quad \text { for some } m \in \mathbb{N}
$$

each of which satisfies the Chern character vanishings formulated in Item (5.1.1) above, cf. [6, Step 2 in proof of Thm. 6.2]. Applying [9, Thm. 6.1] and the Lefschetz hyperplane theorem, [11, Thm. in Sect. II.1.2], we find a smooth complete intersection curve $C \subset X_{\text {reg }}$ such that each $\left.\left(\mathscr{E}_{X_{\text {reg }}}^{(j)}, \theta_{\mathscr{E}_{X \text { reg }}}^{(j)}\right)\right|_{C}$ is stable and such that the natural map

$$
\left(\iota_{C}\right)_{*}: \pi_{1}(C) \rightarrow \pi_{1}\left(X_{\text {reg }}\right)
$$

is surjective. Recall from Remark 3.4 that the Higgs bundle

$$
\left.\left(\mathscr{E}_{X_{\mathrm{reg}}}, \theta_{\mathscr{E}_{\mathrm{X}} \mathrm{reg}}\right)\right|_{C}=\left.\left.\left(\mathscr{E}_{X_{\mathrm{reg}}}^{(1)}, \theta_{\mathscr{E}_{X_{\mathrm{reg}}}}^{(1)}\right)\right|_{C} \oplus \cdots \oplus\left(\mathscr{E}_{X_{\mathrm{reg}}}^{(m)}, \theta_{\mathscr{E}_{X_{\mathrm{reg}}}}^{(m)}\right)\right|_{C}
$$


admits a harmonic bundle structure induced from $\mathbb{E}$ by restriction. The associated flat bundle corresponds to the representation obtained by composing the monodromy representation of $\left(E, \nabla_{\mathbb{E}}\right)$ with $(5.10 .2)$ and is therefore simple. However, via the Simpson correspondence on $C,[37$, Cor. 1.3], this yields a contradiction to the decomposition (5.10.3) of $\left.\left(\mathscr{E}_{X_{\text {reg }}}, \theta_{\mathscr{E}_{X_{\text {reg }}}}\right)\right|_{C}$.

\section{Part II. Applications}

\section{Nonabelian Hodge correspondences for smooth loci}

The existence result for tame and purely imaginary harmonic bundles, Theorem 5.1, yields a nonabelian Hodge correspondence that relates semisimple local systems on the smooth locus of a klt space to polystable Higgs bundles on that locus. As in Simpson's work, this correspondence extends to a correspondence for arbitrary local systems.

\subsection{Nonabelian Hodge correspondence for polystable bundles}

Before formulating the nonabelian Hodge correspondence for polystable bundles in Theorem 6.3 below, we need to specify the appropriate category of bundles. The following definition will be used.

Definition 6.1 Let $X$ be a projective klt space $X$ and $\mathscr{E}_{X_{\text {reg }}}$ be a locally free sheaf on

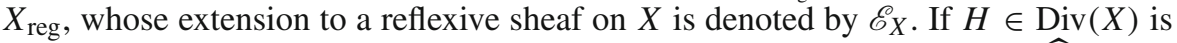
ample, we say that $\mathscr{E}_{X_{\mathrm{reg}}}$ has vanishing $\mathbb{Q}$-Chern classes with respect to $H$ if $\widehat{c h}_{1}\left(\mathscr{E}_{X}\right)$. $[H]^{n-1}=0$ and $\widehat{c h}_{2}\left(\mathscr{E}_{X}\right) \cdot[H]^{n-2}=0$.

As one immediate consequence of the existence result for harmonic structures, Theorem 5.1, we see that a Higgs bundle on the smooth locus of a projective klt space is polystable and has vanishing $\mathbb{Q}$-Chern classes after cutting down with respect to one ample class, iff the same holds for any other ample class. This gives rise to the following fact, which we use to define the relevant category of bundles.

Fact and Definition 6.2 (Category $\mathrm{pHiggs}_{X_{\mathrm{reg}}}$ ). Given a projective klt space $X$ and $a$ locally free Higgs sheaf $\left(\mathscr{E}_{X_{\mathrm{reg}}}, \theta_{\mathscr{E}_{X_{\mathrm{reg}}}}\right)$ on $X_{\mathrm{reg}}$, the following conditions are equivalent.

(6.2.1) There exists an ample $H \in \operatorname{Div}(X)$, such that $\left(\mathscr{E}_{X_{\mathrm{reg}}}, \theta_{\mathscr{E}_{X_{\mathrm{reg}}}}\right)$ is polystable and has vanishing $\mathbb{Q}$-Chern classes with respect to $H$.

(6.2.2) For any ample $H \in \operatorname{Div}(X)$, the Higgs bundle $\left(\mathscr{E}_{X_{\mathrm{reg}}}, \theta_{\mathscr{E}_{X_{\mathrm{reg}}}}\right)$ is polystable and has vanishing $\mathbb{Q}$-Chern classes with respect to $H$.

With their natural morphisms, the Higgs bundle satisfying these conditions form a category, which we denote by $\mathrm{pHiggs}_{X_{\mathrm{reg}}}$.

The nonabelian Hodge correspondence for polystable bundles, which is a direct analogue of [37, Cor. 1.3], is now formulated as follows. 
Theorem 6.3 (Nonabelian Hodge correspondence for $\mathrm{pHiggs}_{X_{\mathrm{reg}}}$ ) Let $X$ be a projective klt space. Then, there exists an equivalence between the category $\mathrm{pHiggs}_{X_{\mathrm{reg}}}$ and the category sLSys $_{X_{\mathrm{reg}}}$ of semisimple local systems on $X_{\mathrm{reg}}$.

For $\left(\mathscr{E}_{X_{\text {reg }}}, \theta_{\mathscr{E}_{X_{\text {reg }}}}\right) \in \mathrm{pHiggs}_{X_{\text {reg }}}$ that are restrictions of polystable Higgs bundles on $X$ with vanishing Chern classes, and for local systems $E_{X_{\mathrm{reg}}} \in \mathrm{sLSys}_{X_{\mathrm{reg}}}$ that are restrictions of local systems on $X$, the correspondence is compatible with the global nonabelian Hodge Correspondence for projective klt spaces found in [9, Sect. 3].

Sketch of proof Starting with a semisimple local system $E_{X_{\mathrm{reg}}}$ on $X_{\mathrm{reg}}$, let $\left(E_{X_{\text {reg }}}, \nabla_{E_{X_{\text {reg }}}}\right)$ be an associated flat bundle. By Theorem 3.13, this bundle admits a tame and purely imaginary harmonic metric that is unique up to flat automorphisms; these preserve the induced decomposition (3.1.1). The uniquely determined associated Higgs bundle $\left(\mathscr{E}_{X_{\text {reg }}}, \theta_{X_{\text {reg }}}\right)$ carries a unique algebraic structure by Remark and Notation 3.9 and is moreover polystable by Lemma 4.7. Its $\mathbb{Q}$-Chern classes vanish by Proposition 3.17 . Assigning $\left(\mathscr{E}_{X_{\text {reg }}}, \theta_{X_{\text {reg }}}\right)$ to $\left(E_{X_{\text {reg }}}, \nabla_{E_{X_{\text {reg }}}}\right)$ defines a functor $\eta_{X_{\text {reg }}}$ from sLSys $X_{\text {reg }}$ to pHiggs $_{X_{\text {reg }}}$. Compatibility with the global nonabelian Hodge Correspondence for projective klt spaces follows from the construction in [9]; cf. especially [9, Prop. 3.10].

We claim that the functor $\eta_{X_{\mathrm{reg}}}$ is an equivalence of categories; for that, we need to check that it is full, faithful and essentially surjective. While essential surjectivity quickly follows from Theorem 5.1, we need to argue a bit more to establish the other two conditions. To this end, let $\gamma: Y \rightarrow X$ be a maximally quasi-étale cover, as provided by [7, Thm. 1.5] and as used in the first part of Sect. 3.2. Denote the Galois group of $\gamma$ by $G$. By the defining property of the maximally quasi-étale cover, the pullback $\gamma^{*}\left(E_{X_{\text {reg }}}\right)$ extends to a $G$-equivariant local system $E_{Y}$ on $Y$. The global nonabelian Hodge Correspondence for projective klt spaces then assigns a G-equivariant, locally free, polystable Higgs bundle $\left(\mathscr{E}_{Y}, \theta_{Y}\right)=\eta_{Y}\left(\gamma^{*}\left(E_{X_{\text {reg }}}\right)\right)$ to $\gamma^{*}\left(E_{X_{\text {reg }}}\right)$, which is seen to coincide with $\gamma^{*}\left(\mathscr{E}_{X_{\text {reg }}}, \theta_{X_{\text {reg }}}\right)=\gamma^{*}\left(\eta_{X_{\text {reg }}}\left(E_{X_{\text {reg }}}\right)\right)$ wherever this makes sense, see Proposition 3.17 as well as Remark and Notation 3.18 and also again [9, Prop. 3.10]. The fact that $\eta_{Y}$ is an equivalence of categories now quickly implies that $\eta_{X_{\text {reg }}}$ is full and faithful as well.

Remark 6.4 It follows from the preceding proof and from Proposition 3.17 that on a maximally quasi-étale cover $Y$, the nonabelian Hodge correspondence for $\mathrm{pHiggs}_{Y_{\mathrm{reg}}}$ coincides with the nonabelian Hodge Correspondence for the projective klt space $Y$ when we apply the natural restriction functors on both sides of the correspondence.

\subsection{Nonabelian Hodge correspondence for semistable bundles}

In direct analogy to Simpson's work, Theorem 6.3 extends to give an equivalence between the category of flat bundles and arbitrary local systems on $X_{\text {reg }}$. The (fairly standard) proof requires a version of the nonabelian Hodge correspondence for a maximally quasi-étale cover [9, Thm. 3.4 and the discussion after Prop. 3.11], Theorem 6.3 above, and the formalities of differential graded categories (DGCs) established in [37, Sect. 3]. The details are left to the reader. 
Fact and Definition 6.5 (Category Higgs ${ }_{X_{\mathrm{reg}}}$ ). Given a projective klt space $X$ and $a$ locally free Higgs sheaf $\left(\mathscr{E}_{X_{\mathrm{reg}}}, \theta_{\mathscr{E}_{X_{\mathrm{reg}}}}\right)$ on $X_{\mathrm{reg}}$, the following conditions are equivalent.

(6.5.1) There exists an ample $H \in \operatorname{Div}(X)$, such that $\left(\mathscr{E}_{X_{\mathrm{reg}}}, \theta_{\mathscr{E}_{X_{\mathrm{reg}}}}\right)$ is semistable and has vanishing $\mathbb{Q}$-Chern classes with respect to $H$.

(6.5.2) For any ample $H \in \operatorname{Div}(X)$, the Higgs bundle $\left(\mathscr{E}_{X_{\mathrm{reg}}}, \theta_{\mathscr{E}_{X_{\mathrm{reg}}}}\right)$ is semistable and has vanishing $\mathbb{Q}$-Chern classes with respect to $H$.

With their natural morphisms, the Higgs bundle satisfying these conditions form a category, which we denote by Higgs ${ }_{X_{\mathrm{reg}}}$.

In analogy to [37, Cor. 3.10], the nonabelian Hodge correspondence for semistable bundles now reads as follows.

Theorem 6.6 (Nonabelian Hodge correspondence for Higgs $_{X_{\mathrm{reg}}}$ ). Let $X$ be a projective klt space. Then, there exists equivalence between the category $\operatorname{Higgs}_{X_{\mathrm{reg}}}$ and the category LSys ${ }_{x_{\mathrm{reg}}}$ of local systems on $X_{\text {reg. }}$.

For $\left(\mathscr{E}_{X_{\mathrm{reg}}}, \theta_{\mathscr{E}_{X_{\mathrm{reg}}}}\right) \in \mathrm{Higgs}_{X_{\mathrm{reg}}}$ that are restrictions of bundles $\left(\mathscr{E}_{X}, \theta_{\mathscr{E}_{X}}\right) \in \operatorname{Higgs}_{X}$ and for local systems $E_{X_{\mathrm{reg}}} \in \mathrm{LSys}_{X_{\mathrm{reg}}}$ that are restrictions of local systems $E_{X} \in$ LSys $_{X}$, the correspondence is compatible with the global nonabelian Hodge Correspondence for projective klt spaces found in [9, Sect. 3].

A statement similar to Remark 6.4 continues to hold for the nonabelian Hodge correspondence for semistable bundles on a maximally quasi-étale cover.

\section{Proof of Theorem 1.5, uniformisation for minimal varieties}

In this section, we prove Theorem 1.5. The strategy of proof in principle follows [8, Prop. 8.2 and 8.3]. The main new difficulty stems from the fact that a general complete intersection surface in a klt surface need not be smooth, but might have finite quotient singularities. We maintain notation and assumptions of Theorem 1.5 throughout.

\section{Step 1: Reduction steps}

Let $\pi: X \rightarrow X_{\text {can }}$ be the birational crepant morphism to the canonical model $X_{\text {can }}$, which is also klt, and whose canonical divisor $K_{X_{\text {can }}}$ is ample, cf. [20, Thm. 3.3].

Claim 7.1 We have the inequality $\widehat{c}_{2}\left(\mathscr{T}_{X_{\text {can }}}\right) \cdot\left[K_{X_{\text {can }}}\right]^{n-2} \leq \widehat{c}_{2}\left(\mathscr{T}_{X}\right) \cdot\left[K_{X}\right]^{n-2}$.

Proof. Let $S_{\text {can }} \subset X_{\text {can }}$ be a surface cut out by general members of the ample linear system $\left|m \cdot K_{X_{\text {can }}}\right|$ for $m \gg 0$, and let $S \subset X$ be its preimage in $X$. Notice that both $S_{\text {can }}$ and $S$ have finite quotient klt singularities. To show Claim 7.1, it is then equivalent to show that

$$
\widehat{c_{2}}\left(\mathscr{T}_{X_{\mathrm{can}}} S_{\mathrm{can}}\right)=\widehat{c}_{2}\left(\mathscr{T}_{X_{\mathrm{can}}}\right) \cdot\left[S_{\mathrm{can}}\right] \leq \widehat{c}_{2}\left(\mathscr{T}_{X}\right) \cdot[S]=\widehat{c}_{2}\left(\left.\mathscr{T}_{X}\right|_{S}\right)
$$

Now the exact sequences of $\mathbb{Q}$-vector bundles

$0 \rightarrow \mathscr{T}_{S_{\text {can }}} \rightarrow \mathscr{T}_{X_{\text {can }}} \mid S_{\text {can }} \rightarrow \mathscr{N}_{S_{\text {can }} / X_{\text {can }}} \rightarrow 0$ and $\left.0 \rightarrow \mathscr{T}_{S} \rightarrow \mathscr{T}_{X}\right|_{S} \rightarrow \mathscr{N}_{S / X} \rightarrow 0$ 
give equalities of $\mathbb{Q}$-Chern numbers,

$$
\begin{aligned}
& \widehat{c_{2}}\left(\left.\mathscr{T}_{X_{\mathrm{can}}}\right|_{S_{\mathrm{can}}}\right)=\widehat{c}_{2}\left(\mathscr{T}_{S_{\mathrm{can}}}\right)+\widehat{c}_{1}\left(\mathscr{T}_{S_{\mathrm{can}}}\right) \cdot c_{1}\left(\mathscr{N}_{S_{\mathrm{can}} / X_{\mathrm{can}}}\right)+\widehat{c}_{2}\left(\mathscr{N}_{S_{\mathrm{can}} / X_{\mathrm{can}}}\right) \\
& \widehat{c}_{2}\left(\left.\mathscr{T}_{X}\right|_{S}\right)=\widehat{c}_{2}\left(\mathscr{T}_{S}\right)+\widehat{c}_{1}\left(\mathscr{T}_{S}\right) \cdot c_{1}\left(\mathscr{N}_{S / X}\right)+\widehat{c}_{2}\left(\mathscr{N}_{S / X}\right) \text {. }
\end{aligned}
$$

There is more that we can say about the summands on the right hand side. First, recall from [24, Thm. 4.2] that $\widehat{c}_{2}\left(\mathscr{T}_{S_{\text {can }}}\right) \leq \widehat{c}_{2}\left(\mathscr{T}_{S}\right)$. Second, recalling that the morphism $S \rightarrow S_{\text {can }}$ is crepant, that $\mathscr{N}_{S_{\text {can }} / X_{\text {can }}}$ is locally free and that $\mathscr{N}_{S / X} \cong\left(\left.\pi\right|_{S}\right)^{*} \mathscr{N}_{S_{\text {can }} / X_{\text {can }}}$, we find equalities,

$\widehat{c}_{1}\left(\mathscr{T}_{S_{\mathrm{can}}}\right) \cdot c_{1}\left(\mathscr{N}_{S_{\mathrm{can}} / X_{\mathrm{can}}}\right)=\widehat{c}_{1}\left(\mathscr{T}_{S}\right) \cdot c_{1}\left(\mathscr{N}_{S(X)}\right)$ and $\widehat{c}_{2}\left(\mathscr{N}_{S_{\mathrm{can}} / X_{\mathrm{can}}}\right)=\widehat{c}_{2}\left(\mathscr{N}_{S / X}\right)$

for $\mathbb{Q}$-Chern numbers on $S_{\text {can }}$ and $S$, respectively.

(Claim 7.1)

As a direct consequence of Claim 7.1 and of the fact that $\pi$ is crepant, we see that equality holds in the $\mathbb{Q}$-Miyaoka-Yau inequality for $X_{\text {can }}$ as well. The variety $X_{\text {can }}$ therefore reproduces the assumptions made in Theorem 1.5, and we may assume for the remainder of the present proof that the divisor $K_{X}$ is ample.

Likewise, if $\gamma: Y \rightarrow X$ is any quasi-étale cover, recall from [20, Prop. 5.20] that $Y$ is again klt. We have remarked in $[8$, Lem. 3.16] that equality holds in the $\mathbb{Q}$-MiyaokaYau inequality for $Y$, too. Replacing $X$ by a suitable maximally quasi-étale cover, [7, Thm. 1.5], we will therefore assume from now on that $X$ is maximally quasi-étale. Our aim is now to show that $X$ is smooth. Once this is established, the main claim will follow from classical uniformisation results of Yau for smooth projective varieties.

\section{Step 2: End of proof}

Consider the locally free sheaf $\mathscr{E}_{X_{\text {reg }}}:=\Omega_{X_{\text {reg }}}^{1} \oplus \mathscr{O}_{X_{\text {reg }}}$ on $X_{\text {reg }}$ and its natural Higgs field

$$
\theta_{\mathscr{E}_{X_{\mathrm{reg}}}}: \Omega_{X_{\mathrm{reg}}}^{1} \oplus \mathscr{O}_{X_{\mathrm{reg}}} \rightarrow\left(\Omega_{X_{\mathrm{reg}}}^{1} \oplus \mathscr{O}_{X_{\mathrm{reg}}}\right) \otimes \Omega_{X_{\mathrm{reg}}}^{1}, \quad(a, b) \mapsto(0,1) \otimes a
$$

Recall from [8, Cor. 7.2] that the Higgs sheaf $\left(\mathscr{E}_{X_{\text {reg }}}, \theta_{\mathscr{E}_{X_{\text {reg }}}}\right)$ is stable with respect to the ample divisor $K_{X}$. Lemma 4.7 then asserts that the endomorphism bundle with its natural Higgs field

$$
\left(\mathscr{F}_{X_{\text {reg }}}, \theta_{\mathscr{F}_{X_{\text {reg }}}}\right):=\mathscr{E} n d\left(\mathscr{E}_{X_{\text {reg }}}, \theta_{\mathscr{E}_{X_{\text {reg }}}}\right)
$$

is polystable with respect to $K_{X}$. Let $\mathscr{F}_{X}$ be the unique extension of $\mathscr{F}_{X_{\text {reg }}}$ as a reflexive sheaf on $X$. By construction, $c_{1}\left(\mathscr{F}_{X}\right)=0$. As an immediate consequence of the assumed Equality (1.5.1) and the calculus of $\mathbb{Q}$-Chern classes, see [8, Lem. 3.18], we obtain the vanishing $\widehat{c h}_{2}\left(\mathscr{F}_{X}\right) \cdot\left[K_{X}\right]^{n-2}=\widehat{c}_{2}\left(\mathscr{F}_{X}\right) \cdot\left[K_{X}\right]^{n-2}=0$. But then Theorem 5.1 implies that

$$
\left(\mathscr{F}_{X_{\text {reg }}}, \theta_{\mathscr{F}_{X_{\text {reg }}}}\right) \in \text { TPI-Higgs } X_{\text {reg }} .
$$


Proposition 3.17 together with the assumption that $X$ is maximally quasi-étale implies that $\mathscr{F}_{X}$ is locally free. Now, as $\mathscr{T}_{X}$ is a direct summand of $\mathscr{F}_{X}=\mathscr{E}_{n d}\left(\Omega_{X}^{[1]} \oplus \mathscr{O}_{X}\right)$, it follows that $\mathscr{T}_{X}$ is locally free. The solution of the Lipman-Zariski conjecture for klt spaces, [5, Thm. 6.1], therefore asserts that $X$ is smooth. Theorem 1.5 now follows from the classical result of Yau, [38, Rem. (iii) on p. 1799].

\section{Positivity in the sheaf of reflexive differentials}

Given a singular ball quotient $X$, we ask for positivity in the sheaf $\Omega_{X}^{[1]}$ of reflexive differentials. In other words, we would like to answer the following question.

Question 8.1 (Positivity for singular ball quotients) Given a morphism $f: Y \rightarrow X$ of projective varieties where $X$ has canonical or klt singularities and where $Y$ is a smooth ball quotient of general type, is $\Omega_{X}^{[1]}$ positive in a suitable sense?

The relevance of Question 8.1 is illustrated by Proposition 8.2 below, which relates positivity to non-existence of rational curves. Moreover, it is motivated by the hyperbolicity statement [8, Cor. 1.4 and Sect. 9.3]. The answer to Question 8.1 turns out to be surprisingly delicate. One the one hand, we show in Sect. 8.2 that sufficiently high symmetric powers $\operatorname{Sym}^{[m]} \Omega_{X}^{[1]}$ are always ample in the sense of Definition 2.4. On the other hand, Sect. 8.3 shows by way of example that even nefness of $\Omega_{X}^{[1]}$ fails in general. In this sense, we have no satisfactory answer to Question 8.1 at present.

\subsection{Consequences of positivity}

As pointed out above, positivity in the singular sheaf $\Omega_{X}^{[1]}$ directly relates to hyperbolicity properties of the underlying variety.

Proposition 8.2 Let $X$ be a projective klt space.

(8.2.1) If $\Omega_{X}^{[1]}$ is nef, then $X$ does not contain rational curves.

(8.2.2) If $\Omega_{X}^{[1]}$ is ample, then $X$ does not contain any curve whose normalisation is of genus one.

The proof of Proposition 8.2 uses the fact that there exists a functorial pull-back functor for reflexive differential forms on klt spaces that agrees with the standard pullback of Kähler differentials wherever that makes sense. We refer to [18, Sect. 5] for a precise reference, and to [10, Sect. 3] for an overview.

Lemma 8.3 (Pull-back of reflexive differential is generically surjective) Let $X$ be a quasi-projective klt space and $Y \subseteq X$ be a smooth subvariety, with inclusion $\iota: Y \rightarrow$ $X$. Then, the pull-back map $d \iota: \iota^{*} \Omega_{X}^{[1]} \rightarrow \Omega_{Y}^{1}$ is generically surjective.

Proof. The problem is local in the étale topology. More precisely, since we aim to prove generic surjectivity, we are free to replace $X$ by any Zariski-open subset that intersects $Y$. Recalling that pull-back of reflexive differentials is functorial, [18, Sect. 5.1] or [21, 
Sect. 14], we may also replace $X$ and $Y$ by finite étale covers. We will now project to $Y$. To be precise, passing to an étale cover if necessary, [5, Prop. 2.26] allows us to assume without loss of generality that there exists a morphism $\Phi: X \rightarrow Y$ whose restriction to $Y$ is isomorphic. Surjectivity of $d \iota$ then comes out of the following diagram,

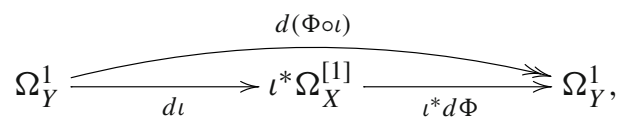

whose commutativity is again due to the functoriality of reflexive pull-back.

Proof of Proposition 8.2 We prove (8.2.2) only. Assume that $\Omega_{X}^{[1]}$ is ample and let $C \subset X$ be any irreducible curve with inclusion $\iota: C \rightarrow X$ and normalisation $\eta: \widetilde{C} \rightarrow C$. Write $f:=\iota \circ \eta$ and notice that Lemma 8.3 implies that the pullback map $d f: f^{[*]} \Omega_{X}^{[1]} \rightarrow \Omega_{\widetilde{C}}^{1}$ is generically surjective. Using that $\widetilde{C}$ is smooth, observe that

$$
f^{[*]} \Omega_{X}^{[1]} \cong f^{*} \Omega_{X}^{[1]} / \text { tor }
$$

as both sheaves are locally free by [31, Cor. on p. 148]. Consequently, $f^{[*]} \Omega_{X}^{[1]}$ is a quotient of the ample sheaf $f^{*} \Omega_{X}^{[1]}$ and therefore itself ample. We conclude that $\operatorname{deg} \Omega_{\widetilde{C}}^{1}>0$, and hence $g(\widetilde{C})>1$, as claimed.

\subsection{Positivity of symmetric differentials}

As pointed out in the introduction, we show that the sheaves of reflexive symmetric differentials of sufficiently high degree are always ample on singular ball quotients.

Proposition 8.4 (Positivity of symmetric differentials for singular ball quotients) Let $f: Y \rightarrow X$ be a quasi-étale cover, where $X$ is projective with klt singularities. If $Y$ is smooth and if $\Omega_{Y}^{1}$ is ample, ${ }^{4}$ then the following will hold.

(8.4.1) The sheaf $f^{[*]} \Omega_{X}^{[1]}$ is ample.

(8.4.2) The sheaf $\operatorname{Sym}^{[m]} \Omega_{X}^{[1]}$ is ample for $m \gg 0$ sufficiently divisible.

Proof. To prove (8.4.1), recall from [18, Thm. 1.3] or [21, Thm. 14.1] that there exists a pull-back morphism for reflexive differential forms, $d f: f^{[*]} \Omega_{X}^{[1]} \rightarrow \Omega_{Y}^{1}$, which is an isomorphism on the big open set where $f$ is étale. Since both sheaves are reflexive, $d f$ is actually an isomorphism, and the first assertion follows.

To prove (8.4.2), recall from [1, Thm. 3.1] that the sheaf $f_{*} \operatorname{Sym}^{m} \Omega_{Y}^{1}$ is ample for all sufficiently large and divisible natural numbers $m$. Over the smooth part of $X$, the projection formula gives

$$
f_{*} \operatorname{Sym}^{m} \Omega_{Y}^{1} \cong f_{*} f^{*} \operatorname{Sym}^{m} \Omega_{X}^{1} \cong \operatorname{Sym}^{m} \Omega_{X}^{1} \oplus\left(\operatorname{Sym}^{m} \Omega_{X}^{1} \otimes f_{*} \mathscr{O}_{Y} / \mathscr{O}_{X}\right) .
$$

\footnotetext{
${ }^{4}$ For instance, this is the case when $Y$ is a smooth ball quotient.
} 
Since $f_{*} \operatorname{Sym}^{m} \Omega_{Y}^{1}$ is reflexive, this implies

$$
f_{*} \operatorname{Sym}^{m} \Omega_{Y}^{1} \cong \operatorname{Sym}^{[m]} \Omega_{X}^{[1]} \oplus\left(\operatorname{Sym}^{m} \Omega_{X}^{1} \otimes f_{*} \mathscr{O}_{Y} / \mathscr{O}_{X}\right)^{* *} .
$$

It follows that $\operatorname{Sym}^{[m]} \Omega_{X}^{[1]}$ is ample as a direct summand of an ample sheaf.

\subsection{Failure of positivity in general}

In spite of the positivity result established in Proposition 8.4, Question 8.1 has a negative answer in general. First examples already exist in dimension two.

Example 8.5 (A klt ball quotient whose reflexive cotangent sheaf is not ample) The example given in [8, Sect. 9.4] shows that there exists a klt ball quotient surface $S$ that is covered by curves whose normalisations are elliptic. As a consequence of Proposition 8.2 above, $\Omega_{S}^{[1]}$ is not ample.

Example 8.6 (A canonical ball quotient whose reflexive cotangent sheaf is not nef) Recall from [3] that there exists a fake projective plane $Y$ that admits an automorphism $\sigma$ of order three. Recall from [29, p. 233] that $Y$ is a smooth ball quotient. The quotient surface $X:=Y /\langle\sigma\rangle$ has been studied by Keum. He proves in [19, Prop. 3.1] that $X$ has exactly three singular points, which are canonical of type $A_{2}$, and that $K_{X}$ is Cartier with $\left[K_{X}\right]^{2}=3$. With this description of $X$ at hand, the following Proposition 8.7 shows that $\Omega_{X}^{[1]}$ cannot possibly be nef.

Proposition 8.7 Let $X$ be a projective surface with non-trivial canonical singularities where $\Omega_{X}^{[1]}$ is nef. If $\left[K_{X}\right]^{2}=3$, then $X$ has exactly one singular point.

Proof. Recall from [20, Thm. 4.20] or [32, p. 347] that $X$ has Du Val surface singularities. Among other things, this implies that $K_{X}$ is Cartier, and that the minimal desingularisation $\pi: \widetilde{X} \rightarrow X$ is crepant, $K_{\widetilde{X}}=\pi^{*} K_{X}$. Denote the singular points of $X$ by $x_{1}, \ldots, x_{k}$. The exceptional set $E \subset \widetilde{X}$ therefore consists of a number (-2)curves arranged in $k$ connected components,

$$
E=\left(E_{1,1} \cup \cdots \cup E_{1, n_{1}}\right) \sqcup \cdots \sqcup\left(E_{k, 1} \cup \cdots \cup E_{1, n_{k}}\right) .
$$

We aim to analyse $\Omega_{X}^{[1]}$ via its reflexive pull-back $\mathscr{E}:=\pi^{[*]} \Omega_{X}^{[1]}$, which is a locally free sheaf on $\widetilde{X}$, cf. [31, Chap. II, Lem. 1.1.10].

Claim 8.8 The sheaf $\mathscr{E}$ is nef. In particular, $c_{1}(\mathscr{E})^{2} \geq 0$.

Proof of Claim 8.8 Consider the natural map from $\mathscr{A}:=\pi^{*} \Omega_{X}^{[1]}$ into its reflexive hull $\mathscr{E}$, which factors as follows

$$
\mathscr{A} \longrightarrow \mathscr{A} / \text { tor } \longrightarrow \mathscr{E}
$$

Nefness of $\mathscr{E}$ can now be concluded using Fact 2.5: as a pull-back of the nef sheaf $\Omega_{X}^{[1]}$, we see that $\mathscr{A}$ nef and then so is its quotient $\mathscr{B}:=\mathscr{A} /$ tor. Since $\widetilde{X}$ is a 
surface, the torsion free sheaf $\mathscr{B}$ is locally free outside of a finite set by [31, Cor. on p. 148], and therefore agrees with $\mathscr{E}$ away from that set. If $C$ is any smooth curve and $\gamma: C \rightarrow X$ any non-constant morphism, the natural map $\gamma^{*} \mathscr{B} \rightarrow \gamma^{*} \mathscr{E}$ is thus generically injective. Using that $C$ is a curve, decompose

$$
\gamma^{*} \mathscr{B}=\underbrace{\left(\gamma^{*} \mathscr{B}\right) / \text { tor }}_{\text {locally free, nef }} \oplus \operatorname{tor}\left(\gamma^{*} \mathscr{B}\right)
$$

The injection $\left(\gamma^{*} \mathscr{B}\right) /$ tor $\hookrightarrow \gamma^{*} \mathscr{E}$ then shows nefness.

$\square($ Claim 8.8)

The existence of $(-2)$-curves in $\widetilde{X}$ shows that $\Omega_{\widetilde{X}}^{1}$ is not nef. Claim 8.8 thus shows that the canonical pull-back morphism for reflexive differentials, $d \pi: \mathscr{E} \rightarrow \Omega_{\widetilde{X}}^{1}$, which exists by [5, Thm. 4.3] or [21, Thm. 1.9], is not isomorphic at the generic point of any such curve. Somewhat more quantitatively, we find strictly positive $\lambda_{i, j} \in \mathbb{N}$ such that

$$
\operatorname{det} \mathscr{E} \cong \omega_{\widetilde{X}}\left(-\sum_{i, j} \lambda_{i, j} \cdot E_{i, j}\right)=\omega_{\widetilde{X}}\left(-\sum_{i} F_{i}\right), \quad \text { for } F_{i}:=\sum_{j} \lambda_{i, j} \cdot E_{i, j}
$$

Observe that $\left[F_{i}\right] \cdot\left[F_{j}\right]=0$ for $i \neq j$ and that $\left[F_{i}\right]^{2}<0$ for all $i$. Better still, since $\left[F_{i}\right] \cdot\left[K_{\tilde{X}}\right]=0$, Riemann-Roch on $\widetilde{X}$ asserts that the numbers $\left[F_{i}\right]^{2}$ are all even. But then

$$
c_{1}(\mathscr{E})^{2}=\left[\pi^{*} K_{X}-\sum_{i=1}^{k} F_{i}\right]^{2}=\underbrace{\left[K_{X}\right]^{2}}_{=3}+\sum_{i=1}^{k} \underbrace{\left[F_{i}\right]^{2}}_{\leq-2, \text { even }} \stackrel{\text { Claim } 8.8}{\geq} 0 .
$$

We obtain that $k=1$, as claimed.

Acknowledgements We would like to thank numerous colleagues for discussions, including Daniel Barlet, Oliver Bräunling, Philippe Eyssidieux, Jochen Heinloth, Andreas Höring, Annette Huber, Shane Kelly, Jong-Hae Keum, Adrian Langer and Jörg Schürmann. We also thank the anonymous referee for helpful suggestions for improvement.

Open Access This article is distributed under the terms of the Creative Commons Attribution 4.0 International License (http://creativecommons.org/licenses/by/4.0/), which permits unrestricted use, distribution, and reproduction in any medium, provided you give appropriate credit to the original author(s) and the source, provide a link to the Creative Commons license, and indicate if changes were made.

\section{References}

1. Ancona, V.: Faisceaux amples sur les espaces analytiques. Trans. Am. Math. Soc. 274(1), 89-100 (1982). https://doi.org/10.2307/1999498

2. Beltrametti, M.C., Sommese, A.J.: The adjunction theory of complex projective varieties. In: De Gruyter Expositions in Mathematics, vol. 16. Walter de Gruyter \& Co., Berlin (1995). https://doi.org/ $10.1515 / 9783110871746$

3. Cartwright, D.I., Steger, T.: Enumeration of the 50 fake projective planes. C. R. Math. Acad. Sci. Paris 348(1-2), 11-13 (2010). https://doi.org/10.1016/j.crma.2009.11.016

4. Fulton, W.: Intersection Theory, volume 2 of Ergebnisse der Mathematik und ihrer Grenzgebiete. 3. Folge. A Series of Modern Surveys in Mathematics [Results in Mathematics and Related Areas. 3rd 
Series. A Series of Modern Surveys in Mathematics], 2nd edn. Springer, Berlin (1998). https://doi.org/ 10.1007/978-1-4612-1700-8

5. Greb, D., Kebekus, S., Kovács, S.J., Peternell, T.: Differential forms on log canonical spaces. Inst. Hautes Études Sci. Publ. Math. 114(1), 87-169 (2011). https://doi.org/10.1007/s10240-011-0036-0. An extended version with additional graphics is available as arXiv:1003.2913

6. Greb, D., Kebekus, S., Peternell, T.: Movable curves and semistable sheaves. Int. Math. Res. Not. 2016(2), 536-570 (2016). https://doi.org/10.1093/imrn/rnv126. Preprint arXiv:1408.4308

7. Greb, D., Kebekus, S., Peternell, T.: Étale fundamental groups of Kawamata log terminal spaces, flat sheaves, and quotients of abelian varieties. Duke Math. J. 165(10), 1965-2004 (2016). https://doi.org/ 10.1215/00127094-3450859. Preprint arXiv:1307.5718

8. Greb, D., Kebekus, S., Peternell, T., Taji, B.: The Miyaoka-Yau inequality and uniformisation of canonical models. Preprint arXiv:1511.08822. To appear in the Annales scientifiques de l'École normale supérieure

9. Greb, D., Kebekus, S., Peternell, T., Taji, B.: Nonabelian Hodge theory for klt spaces and descent theorems for vector bundles. Compos. Math. 155(2), 289-323 (2019). https://doi.org/10.1112/ S0010437X18007923. Preprint arXiv:1711.08159

10. Greb, D., Kebekus, S., Taji, B..: Uniformisation of higher-dimensional varieties. In: de Fernex, T., Hassett, B., Mustaţă, M., Olsson, M., Popa, M., Thomas, R. (eds.) Algebraic Geometry: Salt Lake City 2015, volume 1 of Proceedings of Symposia in Pure Mathematics, pp. 277-308. American Mathematical Society, American Mathematical Society (2018). Preprint arXiv:1608.06644

11. Goresky, M., MacPherson, R.D.: Stratified Morse theory, volume 14 of Ergebnisse der Mathematik und ihrer Grenzgebiete (3) [Results in Mathematics and Related Areas (3)]. Springer, Berlin (1988). https://doi.org/10.1007/978-3-642-71714-7

12. Grothendieck, A.: Techniques de construction en géométrie analytique. V. Fibrés vectoriels, fibrés projectifs, fibrés en drapeaux. In Séminaire Henri Cartan, volume 13, chapter Exp. $\mathrm{n}^{\circ} 12$, pages 1-15. Secrétariat mathématique, Paris, 1960-1961. Revised in collaboration with Jean Dieudonné. numdam.SHC-1960-1961-13-1-A8-0

13. Grothendieck, A.: Représentations linéaires et compactification profinie des groupes discrets. Manuscr. Math. 2, 375-396 (1970). https://doi.org/10.1007/BF01719593

14. Guenancia, H., Taji, B.: Orbifold stability and Miyaoka-Yau inequality for minimal pairs. Preprint arXiv:1611.05981 (2016)

15. Hartshorne, R.: Algebraic geometry. Springer, New York, Graduate Texts in Mathematics. No. 52, (1977). https://doi.org/10.1007/978-1-4757-3849-0

16. Huybrechts, D., Lehn, M.: The geometry of moduli spaces of sheaves. In: Cambridge Mathematical Library. Cambridge University Press, Cambridge (2010). https://doi.org/10.1017/ CBO9780511711985

17. Jost, J., Zuo, K.: Harmonic maps of infinite energy and rigidity results for representations of fundamental groups of quasiprojective varieties. J. Differ. Geom. 47, 469-503 (1997). euclid.jdg/1214460547

18. Kebekus, S.: Pull-back morphisms for reflexive differential forms. Adv. Math. 245, 78-112 (2013). https://doi.org/10.1016/j.aim.2013.06.013. Preprint arXiv:1210.3255

19. Keum, J.-H.: Quotients of fake projective planes. Geom. Topol. 12(4), 2497-2515 (2008). https://doi. org/10.2140/gt.2008.12.2497

20. Kollár, J., Mori, S.: Birational geometry of algebraic varieties. In: Cambridge Tracts in Mathematics, vol. 134. Cambridge University Press, Cambridge (1998). With the collaboration of C. H. Clemens and A. Corti, Translated from the 1998 Japanese original. https://doi.org/10.1017/CBO9780511662560

21. Kebekus, S., Schnell, C.: Extending holomorphic forms from the regular locus of a complex space to a resolution of singularities. Preprint arXiv:1811.03644 (2018)

22. Le Potier, J.: Fibrés de Higgs et systémes locaux. Astérisque, (201-203):Exp. No. 737, 221-268 (1992), 1991. Séminaire Bourbaki, Vol. 1990/91

23. Lu, S.S.-Y., Taji, B.: A characterization of finite quotients of Abelian varieties. IMRN 2018(1), 292-319 (2014). https://doi.org/10.1093/imrn/rnw251. Preprint arXiv:1410.0063

24. Megyesi, G.: Generalisation of the Bogomolov-Miyaoka-Yau inequality to singular surfaces. Proc. Lond. Math. Soc. (3) 78(2), 241-282 (1999). https://doi.org/10.1112/S0024611599001719

25. Mochizuki, T.: Kobayashi-Hitchin correspondence for tame harmonic bundles and an application. Astérisque 309, viii+117 (2006)

26. Mochizuki, T.: Asymptotic behaviour of tame harmonic bundles and an application to pure twistor D-modules. I. Mem. Am. Math. Soc. 185(869), xii+324 (2007). https://doi.org/10.1090/memo/0869 
27. Mochizuki, T.: Asymptotic behaviour of tame harmonic bundles and an application to pure twistor D-modules. II. Mem. Am. Math. Soc. 185(870), xii+565 (2007). https://doi.org/10.1090/memo/0870

28. Mehta, V.B., Ramanathan, A.: Restriction of stable sheaves and representations of the fundamental group. Invent. Math. 77(1), 163-172 (1984). https://doi.org/10.1007/BF01389140

29. Mumford, D.: An algebraic surface with $K$ ample, $\left(K^{2}\right)=9, p_{g}=q=0$. Am. J. Math. 101(1), 233-244 (1979). https://doi.org/10.2307/2373947

30. Mumford, D.: Towards an enumerative geometry of the moduli space of curves. In: Arithmetic and Geometry, vol. II, volume 36 of Progr. Math., pp. 271-328. Birkhäuser Boston (1983). https://doi.org/ 10.1007/987-1-4757-9286-7-12

31. Okonek, C., Schneider, M., Spindler, H.: Vector bundles on complex projective spaces. In: Progress in Mathematics, vol. 3. Birkhäuse, Boston, Mass (1980)

32. Reid, M.: Young person's guide to canonical singularities. In: Algebraic Geometry, Bowdoin, 1985 (Brunswick, Maine, 1985), volume 46 of Proc. Sympos. Pure Math., pp. 345-414. American Mathematical Society, Providence (1987)

33. Sabbah, C.: Théorie de Hodge et correspondance de Hitchin-Kobayashi sauvages (d'après T. Mochizuki). Astérisque, (352):Exp. No. 1050, viii, 205-241 (2013). Séminaire Bourbaki. Vol. 2011/2012. Exposés 1043-1058

34. Shepherd-Barron, N.I., Wilson, P.M.H.: Singular threefolds with numerically trivial first and second Chern classes. J. Algebraic Geom. 3(2), 265-281 (1994)

35. Serre, J.-P.: Prolongement de faisceaux analytiques cohérents. Ann. Inst. Fourier (Grenoble) 16(fasc. 1), 363-374 (1966)

36. Simpson, C.T.: Constructing variations of Hodge structure using Yang-Mills theory and applications to uniformization. J. Am. Math. Soc. 1(4), 867-918 (1988). https://doi.org/10.1090/S0894-0347-19880944577-9

37. Simpson, C.T.: Higgs bundles and local systems. Inst. Hautes Études Sci. Publ. Math. 75, 5-95 (1992). https://doi.org/10.1007/BF02699491. numdam.PMIHES-1992-75-5-0

38. Yau, S.-T.: Calabi's conjecture and some new results in algebraic geometry. Proc. Natl. Acad. Sci. USA 74(5), 1798-1799 (1977)

Publisher's Note Springer Nature remains neutral with regard to jurisdictional claims in published maps and institutional affiliations.

\section{Affiliations}

\section{Daniel Greb ${ }^{1} \cdot$ Stefan Kebekus ${ }^{2,3} \cdot$ Thomas Peternell $^{4} \cdot$ Behrouz Taji $^{5}$}

$凶$ Daniel Greb

daniel.greb@uni-due.de

https://www.esaga.uni-due.de/daniel.greb

Stefan Kebekus

stefan.kebekus@math.uni-freiburg.de

https://cplx.vm.uni-freiburg.de

Thomas Peternell

thomas.peternell@uni-bayreuth.de

http://www.komplexe-analysis.uni-bayreuth.de

Behrouz Taji

behrouz.taji@sydney.edu.au

http://www.maths.usyd.edu.au/u/behrouzt/

1 Essener Seminar für Algebraische Geometrie und Arithmetik, Fakultät für Mathematik, Universität Duisburg-Essen, 45117 Essen, Germany

2 Mathematisches Institut, Albert-Ludwigs-Universität Freiburg, Ernst-Zermelo-Strasse 1, 79104 Freiburg im Breisgau, Germany 
3 Freiburg Institute for Advanced Studies (FRIAS), Freiburg im Breisgau, Germany

4 Mathematisches Institut, Universität Bayreuth, 95440 Bayreuth, Germany

5 School of Mathematics and Statistics F07, The University of Sydney, Camperdown, NSW 2006, Australia 